\title{
ON THE MONODROMY OF THE MODULI SPACE OF CALABI-YAU THREEFOLDS COMING FROM EIGHT PLANES IN $\mathbb{P}^{3}$
}

\author{
RALF GERKMANN, MAO SHENG ${ }^{\dagger}$, DUCO VAN STRATEN, AND KANG ZUO
}

To the memory of Eckart Viehweg

\begin{abstract}
It is a fundamental problem in geometry to decide which moduli spaces of polarized algebraic varieties are embedded by their period maps as Zariski open subsets of locally Hermitian symmetric domains. In the present work we prove that the moduli space of Calabi-Yau threefolds coming from eight planes in $\mathbb{P}^{3}$ does not have this property. We show furthermore that the monodromy group of a good family is Zariski dense in the corresponding symplectic group. Moreover, we study a natural sublocus which we call hyperelliptic locus, over which the variation of Hodge structures is naturally isomorphic to wedge product of a variation of Hodge structures of weight one. It turns out the hyperelliptic locus does not extend to a Shimura subvariety of type III (Siegel space) within the moduli space. Besides general Hodge theory, representation theory and computational commutative algebra, one of the proofs depends on a new result on the tensor product decomposition of complex polarized variations of Hodge structures.
\end{abstract}

\section{INTRODUCTION}

A fundamental result of E. Viehweg [30] states that for any polarized algebraic variety the coarse moduli space $\mathfrak{M}$ exists as a quasi-projective variety. It is of great interest to characterize those cases in which $\mathfrak{M}$ is a locally Hermitian symmetric variety. It has been shown in [31], [32], [18 that Arakelov-type equalities lead to sufficient conditions for this to happen. In this paper we describe the techniques of characteristic varieties that leads to necessary conditions that can be checked by a straightforward calculation in concrete examples. This leads to a computational tool that we apply to the moduli space of double octics ramified over an arrangement of eight planes in $\mathbb{P}^{3}$.

As over a coarse moduli space $\mathfrak{M}$ there usually does not exist a family, we use the following weaker notion. We say that a proper smooth map $f: \mathcal{X} \rightarrow S$ over a smooth connected base $S$ is a good family for $\mathfrak{M}$, if the moduli map $S \rightarrow \mathfrak{M}$ of $f$ is dominant and generically finite. The local system $\mathbb{V}:=\left(R^{n} f_{*} \mathbb{Q}_{\mathcal{X}}\right)_{p r}$ of primitive cohomomogies has the structure of a weight $n$ polarized variation of $\mathbb{Q}$ Hodge structures, in short $\mathbb{Q}$-PVHS, over $S$. Recall that this means, among other

\footnotetext{
${ }^{1}$ This work was supported by the SFB/TR 45 Periods, Moduli Spaces and Arithmetic of Algebraic Varieties of the DFG (German Research Foundation).

${ }^{\dagger}$ The second named author is supported by a postdoctoral fellowship in the East China Normal University and is also partially supported by the Program for Changjiang Scholars and Innovative Research Team in University.
} 
things, that there is an Hodge filtration $\mathcal{F}^{\bullet}$ on the vector bundle $\mathcal{V}:=\mathbb{V} \otimes \mathcal{O}_{S}$ with flat connection $\nabla$ for which Griffiths transversality $\nabla \mathcal{F}^{p} \subset \mathcal{F}^{p-1} \otimes \Omega_{S}^{1}$ holds. The associated graded object $(E, \theta)=\left(g r_{F} \mathcal{V}, g r_{F} \nabla\right)=\left(\sum_{p+q=n} E^{p, q}, \oplus_{p+q=n} \theta^{p, q}\right)$ where $E^{p, q}:=\mathcal{F}^{p} / \mathcal{F}^{p-1}$ are the Hodge-bundles and $\theta$ is induced by $\nabla$ is called the associated Higgs-bundle. In general, we call a PVHS $\mathbb{V}$ over $S$ of Calabi-Yau type (CY-type) if rank $E^{n, 0}=1$ and the morphism of vector bundles

$$
\mathcal{T}_{S} \longrightarrow \operatorname{Hom}\left(E^{n, 0}, E^{n-1,1}\right)
$$

induced by $\theta^{n, 0}: E^{n, 0} \rightarrow E^{n-1,1} \otimes \Omega_{S}^{1}$ is an isomorphism at the generic point. It follows from the Bogomolov-Tian-Todorov theorem on the unobstructedness of the infinitesimal deformations of a Calabi-Yau manifold $X$, that for a good family the map $\theta^{n, 0}$ is naturally identified with the Kodaira-Spencer isomorphism, so in such a situation we obtain a PVHS of CY-type.

In this paper we study a particular example of an interesting family of Calabi-Yau threefolds. An arrangement $\mathfrak{A}$ of eight planes in general position in $\mathbb{P}^{3}$ determines a double cover $X$, which is a Calabi-Yau variety with singularities along 28 lines. A resolution $\tilde{X}$ of such a double octic has $\operatorname{dim} H^{3}(\tilde{X})=20$ and carries a weight 3 polarized Hodge structure with Hodge numbers $(1,9,9,1)$. If we vary the arrangement $\mathfrak{A}$ in a good family, we obtain an irreducible weight three $\mathbb{Q}$-PVHS $\mathbb{V}$ of CY-type over a smooth 9 dimensional base $S$. We will show several theorems about $\mathbb{V}$.

Theorem 1.1. $\mathbb{V}$ does not factor canonically.

By this we mean the following. Associated to a Hermitian symmetric domain $D_{0}=G_{0} / K_{0}$ there is a special PVHS $\mathbb{W}$ on $D_{0}$ coming from the representation $\rho_{\text {can }}: G_{0} \rightarrow G L(W)$, which is called the canonical PVHS by B. Gross. We say that $\mathbb{V}$ factors canonically if the period map of $\mathbb{V}$ factors through the one determined by certain canonical PVHS $\left(D_{0}, \rho_{c a n}\right)$. For a precise definition see $\S 3$.

A way to exclude this happening consists of picking an appropriate point $s \in S$, compute an appropriate characteristic subvariety of $\mathbb{V}$ in $\mathbb{P}\left(\mathcal{T}_{S, s}\right)$ and compare it with the corresponding object for $\mathbb{W}$. If these varieties are not isomorphic we are done.

Our example is a member of a well-known infinite series of Calabi-Yau $n$-folds coming from double covers of generic arrangements of $2 n+2$ hyperplanes in $\mathbb{P}^{n}$. For $n=1$ one has the classical theory of four points in $\mathbb{P}^{1}$ and the associated elliptic curves and their modulus. The paper [17] was devoted to $n=2$ case. Here we have K3-surfaces that are double covers of six lines in the plane. In [17] it was shown among other things that in this case there is a natural good family whose associated weight 2 PVHS factors canonically. It was asked by I. Dolgachev (see [2]) if the $n \geq 3$ cases are canonical with respect to $\left(D_{n, n}^{I}, \rho_{\text {can }}\right)$, where $\rho_{\text {can }}: S U(n, n) \stackrel{\wedge^{n}}{\longrightarrow} S p\left(\left(\begin{array}{c}2 n \\ n\end{array}\right), \mathbb{R}\right)$ is the indicated representation of real Lie groups. Motivated by this question, it has been checked in [26] that the primitive 
Hodge numbers of CY $n$-folds are exactly the same as predicted by Dolgachev for all $n \in \mathbb{N}$.

In the pioneering work [25] a result similar to Theorem 1.1] for more general moduli spaces of configurations was given, but the methods used there are completely different from ours. Our method is based on classical Hodge theory (see for example [10]) and can be applied to many other concrete moduli spaces, for example the moduli spaces of the Calabi-Yau varieties in toric varieties. Moreover, we hope to extend the present work to the $n \geq 4$ cases.

Using Theorem 1.1 we will prove the

Theorem 1.2. Let $s \in S$ be a base point and let

$$
\tau: \pi_{1}(S, s) \rightarrow S p(20, \mathbb{Q})
$$

be the monodromy representation associated to $\mathbb{V}$. Then the image of $\tau$ is Zariski dense in $\operatorname{Sp}(20, \mathbb{R})$.

Using results of C. Schoen and P. Deligne the above theorem implies:

Corollary 1.3. The special Mumford-Tate group of a general member in $\mathfrak{M}_{C Y}$ is $\operatorname{Sp}(20, \mathbb{Q})$.

However, there exists an interesting subvariety of $\mathfrak{M}_{C Y}$ where the special MumfordTate groups are proper subgroups of $S p(20, \mathbb{Q})$. Generalizing a construction from [17, we define a five-dimensional subvariety $\mathfrak{H}_{C Y} \subset \mathfrak{M}_{C Y}$ that we call the hyperelliptic locus. Over it, the Hodge structure is isomorphic to $\wedge^{3}$ of a $H^{1}(C)$, where $C$ is a hyperelliptic curve of genus three. It is natural to ask if this decomposition can be extended to a larger variety $\mathfrak{H}$ that contains $\mathfrak{H}_{C Y}$. Using a calculation of characteristic subvarieties we arrive at a negative answer.

Theorem 1.4. Let $\mathfrak{H}_{C Y}$ be the hyperelliptic locus of $\mathfrak{M}_{C Y}$ and $\mathfrak{H}$ be any subvariety of $\mathfrak{M}_{C Y}$ which strictly contains $\mathfrak{H}_{C Y}$. Let $f: \mathcal{X} \rightarrow S$ be a good family for $\mathfrak{M}_{C Y}$ whose moduli map $S \rightarrow \mathfrak{M}_{C Y}$ is dominant over $\mathfrak{H}$. Then the restriction of $\mathbb{V}$ to the inverse image of $\mathfrak{H}$ does not factor through $\left(D_{3}^{I I I}, \wedge^{3}\right)$.

As a corollary we have the following

Corollary 1.5. The special Mumford-Tate group of the Calabi-Yau threefolds in $\mathfrak{H}_{C Y}$ is a subgroup of $S p(6, \mathbb{Q})$. Furthermore $\mathfrak{H}_{C Y}$ is maximal with this property. That is, for any irreducible subvariety $\mathfrak{H}$ of $\mathfrak{M}_{C Y}$ which strictly contains $\mathfrak{H}_{C Y}$, the special Mumford-Tate group of a general closed point in $\mathfrak{H}$ is not contained in $\operatorname{Sp}(6, \mathbb{Q})$.

The proof of Theorem 1.2 relies on new results of Hodge-theoretical nature. There is Theorem 1.6 on the tensor product decomposition of $\mathbb{C}$-PVHS, parallel to the direct sum decomposition of $\mathbb{C}$-PVHS due to P. Deligne (cf. 55). Let $\bar{S}$ denote a projective manifold, $Z$ a simple divisor with normal crossing and $S=\bar{S} \backslash Z$. Let $\mathbb{V}$ denote an irreducible $\mathbb{C}$-PVHS over $S$ with quasi-unipotent local monodromy around each component of $Z$. Fix a base point $s \in S$ let

$$
\rho: \pi_{1}(S, s) \longrightarrow \mathrm{GL}\left(\mathbb{V}_{s}\right)
$$


denote the representation of the fundamental group associated to the underlying local system of $\mathbb{C}$-vector spaces, where $\mathbb{V}_{s}$ denotes the fibre of $\mathbb{V}$ over $s$. Let $G$ be the Zariski closure of the image of $\rho$ inside $\mathrm{GL}\left(\mathbb{V}_{s}\right)$. Assume that $G$ decomposes into a direct product $G_{1} \times \cdots \times G_{k}$ of simple Lie groups. Then according to Schur's lemma, we obtain a decomposition of local systems $\mathbb{V} \cong \mathbb{V}_{1} \otimes \cdots \otimes \mathbb{V}_{k}$.

Theorem 1.6. Each local system $\mathbb{V}_{i}$ admits the structure of a $\mathbb{C}$-PVHS such that the induced $\mathbb{C}-P V H S$ on the tensor product $\mathbb{V}_{1} \otimes \cdots \otimes \mathbb{V}_{k}$ coincides with the given $\mathbb{C}$-PVHS on $\mathbb{V}$.

The proof of the theorem is independent of the other results in this paper. We expect the result to be useful in other situations. In this article it helps to prove the following classification result.

Theorem 1.7. Let $S$ be a smooth quasi-projective algebraic variety and $\mathbb{V}$ be an weight $3 \mathbb{Z}-P V H S$ over $S$ which is irreducible over $\mathbb{C}$ and of quasi-unipotent local monodromies. If the Hodge numbers of $\mathbb{V}$ are $(1,9,9,1)$, then after a possible finite étale base change the connected component of the real Zariski closure of the monodromy group of $\mathbb{V}$ is one of the following:
(A) $S U(1,1) \times S O_{0}(2,8)$,
(B) $S U(3,3)$,
(C) $\operatorname{Sp}(6, \mathbb{R})$,
(D) $S p(20, \mathbb{R})$.

Using this theorem and Theorem 1.1, 1.4 to exclude cases (A), (B) and (C) one easily obtains Theorem 1.2 .

\section{Two Calabi-Yau Threefolds}

Consider an arrangement $\mathfrak{A}=\left(H_{1}, \ldots, H_{8}\right)$ of eight planes in $\mathbb{P}^{3}$. Such an arrangement can be given by a matrix $A \in M(8 \times 4, \mathbb{C})$, the $i$-th row corresponding to the defining equation

$$
\sum_{j=1}^{4} a_{i j} x_{j}=0
$$

of the hyperplane $H_{i}$. We say that $\mathfrak{A}$ is in general position if no four of the planes intersect in a point. In terms of the matrix $A$ this means that each $(4 \times 4)$-minor is non-zero. We now describe two closely related Calabi-Yau threefolds associated to such an arrangement $\mathfrak{A}$ in general position.

2.1. The double octic. The planes of the arrangement $\mathfrak{A}$ determine a divisor $R=\sum_{i=1}^{8} H_{i}$ on $\mathbb{P}^{3}$. As the degree of $R$ is even and the Picard group of $\mathbb{P}^{3}$ has no torsion, there exists a unique double cover $\pi: X \rightarrow \mathbb{P}^{3}$ that ramifies over $R$. The singular locus of such a double octic $X$ is precisely the preimage of the singular locus of $R$. Its irreducible components are given by the lines $H_{i j}=H_{i} \cap H_{j}$ for $1 \leq i<j \leq 8$. We fix an ordering of the index set $I=\left\{(i, j) \in \mathbb{N}^{2} \mid 1 \leq i<\right.$ $j \leq 8\}$ and let $\phi: \widetilde{\mathbb{P}}^{3} \rightarrow \mathbb{P}^{3}$ denote the composition of blow-ups whose centers 
are the strict transforms of $H_{i j}$, taken in the chosen order. The fibre product $\widetilde{X}:=X \times_{\mathbb{P}^{3}} \widetilde{\mathbb{P}}^{3}$ sits in a commutative diagram

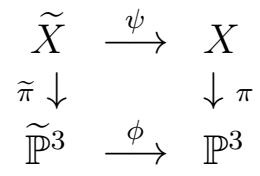

In case we start with an arrangement in general position, the variety $\widetilde{X}$ thus obtained is a smooth Calabi-Yau threefold. Note however that a different ordering of $I$ yields a different birational minimal model of the singular variety $X$.

Lemma 2.1. The space of infinitesimal deformations of $\widetilde{X}$ is naturally isomorphic to the space of infinitesimal deformations of $\mathfrak{A}$.

Proof. This follows from the description of infinitesimal deformation space of a double covers obtained in [3]. We let $\mathcal{L}(\operatorname{resp} . \tilde{\mathcal{L}})$ be the line bundles on $\mathbb{P}^{3}$ (resp. $\left.\widetilde{\mathbb{P}}^{3}\right)$ in the decomposition

$$
\pi_{*} \mathcal{O}_{X}=\mathcal{O}_{\mathbb{P}^{3}} \oplus \mathcal{L}^{-1}\left(\text { resp. } \pi_{*} \mathcal{O}_{\widetilde{X}}=\mathcal{O}_{\widetilde{\mathbb{P}} 3} \oplus \tilde{\mathcal{L}}^{-1}\right) .
$$

It satisfies $\mathcal{L}^{\otimes 2} \simeq \mathcal{O}_{\mathbb{P}^{3}}(R)$ (resp. $\tilde{\mathcal{L}}^{\otimes 2} \simeq \mathcal{O}_{\widetilde{\mathbb{P}}^{3}}(\widetilde{R})$ for $\widetilde{R}$ the strict transform of $R$ under $\phi)$. One has the decomposition of tangent sheaf

$$
\pi_{*} \mathcal{T}_{\widetilde{X}}=\mathcal{T}_{\mathbb{P}^{3}}(-\log \widetilde{R}) \oplus \mathcal{T}_{\widetilde{\mathbb{P}} 3} \otimes \tilde{\mathcal{L}}^{-1} .
$$

It follows that one has natural isomorphism (see also Prop. 2.1 [3])

$$
H^{1}\left(\mathcal{T}_{\widetilde{X}}\right) \simeq H^{1}\left(\mathcal{T}_{\mathbb{P} 3}(-\log \widetilde{R})\right) \oplus H^{1}\left(\mathcal{T}_{\widetilde{\mathbb{P}}^{3}} \otimes \tilde{\mathcal{L}}^{-1}\right)
$$

By Corollary 4.3 in [3], the space $H^{1}\left(\mathcal{T}_{\mathbb{P}^{3}}(-\log \widetilde{R})\right)$ is naturally isomorphic to the space of equisingular deformations of $R$ in $\mathbb{P}^{3}$. Since $R$ is a divisor with normal crossings, this space is isomorphic to the space of infinitesimal deformations of the arrangement $\mathfrak{A}$ in $\mathbb{P}^{3}$. Furthermore, the space of transverse deformation $H^{1}\left(\mathcal{T}_{\widetilde{\mathbb{P}} 3} \otimes \tilde{\mathcal{L}}^{-1}\right)$ has dimension (after Prop. $5.1[3]$ )

$$
\begin{aligned}
h^{1}\left(\mathcal{T}_{\widetilde{\mathbb{P}}^{3}} \otimes \tilde{\mathcal{L}}^{-1}\right) & =h^{1}\left(\mathcal{T}_{\mathbb{P}^{3}} \otimes \mathcal{L}^{-1}\right)+\sum_{(i, j) \in I} h^{0}\left(K_{H_{i j}}\right) \\
& =h^{1}\left(\mathcal{T}_{\mathbb{P}^{3}} \otimes \mathcal{O}(-3)\right)+\sum_{(i, j) \in I} h^{0}\left(K_{\mathbb{P}^{1}}\right) \\
& =0
\end{aligned}
$$

where the vanishing of the first summand follows from Bott's vanishing theorem on homogenous vector bundles over the projective spaces. The lemma thus follows.

From now on we let $\mathfrak{M}_{A R}$ denote the moduli space of arrangements $\mathfrak{A}$ of eight planes in $\mathbb{P}^{3}$ in general position, and let $\mathfrak{M}_{C Y}$ denote the moduli space of $\widetilde{X}$.

Corollary 2.2. The moduli map $\mathfrak{M}_{A R} \rightarrow \mathfrak{M}_{C Y}$ is étale. 
There are many ways to construct a good family for $\mathfrak{M}_{A R}$. Here is one. Let $\mathfrak{A}$ be an arrangement in general position. The moduli point of $\mathfrak{A}$ in $\mathfrak{M}_{A R}$ can be uniquely represented by the matrix $A$ of the form:

$$
\left(\begin{array}{llllllll}
1 & 0 & 0 & 0 & 1 & 1 & 1 & 1 \\
0 & 1 & 0 & 0 & 1 & * & * & * \\
0 & 0 & 1 & 0 & 1 & * & * & * \\
0 & 0 & 0 & 1 & 1 & * & * & *
\end{array}\right)^{t}
$$

Conversely a matrix $A$ in the above form whose all $4 \times 4$ minors are nonzero represents an arrangement $\mathfrak{A}$ in general position. Thus $\mathfrak{M}_{A R}$ can be realized as an open subvariety of the affine space $\mathbb{C}^{9}$ and it admits a natural good family $f_{0}: \mathcal{X} \rightarrow \mathfrak{M}_{A R}$, where $\mathcal{X}$ is obtained by simultaneous resolution of the singular double octic over $\mathfrak{M}_{A R}$. Note also that a good family for $\mathfrak{M}_{A R}$ gives rise to a good family for $\mathfrak{M}_{C Y}$.

Remark 2.3. This construction and the above lemma can actually be generalized to arrangements of $2 n+2$ hyperplanes in $\mathbb{P}^{n}$. It yields a moduli space of smooth CY $n$-folds whose primitive Hodge numbers are given by $h_{p r}^{p, n-p}(\widetilde{X})=\left(\begin{array}{l}n \\ p\end{array}\right)^{2}$. For details, we refer to [26].

Now we proceed with the construction of another Calabi-Yau threefold $Y$ for a given arrangement $\mathfrak{A}$.

2.2. The Kummer cover. Let $A=\left(a_{i j}\right)$ denote a $(8 \times 4)$-matrix associated to $\mathfrak{A}$ as described above. Furthermore let $B=\left(b_{i j}\right)$ denote a matrix in $M(4 \times 8, \mathbb{C})$ such that the sequence

$$
0 \longrightarrow \mathbb{C}^{4} \stackrel{A}{\longrightarrow} \mathbb{C}^{8} \stackrel{B}{\longrightarrow} \mathbb{C}^{4} \longrightarrow 0
$$

is exact. We let $Y$ denote the complete intersection of the four quadrics in $\mathbb{P}^{7}$ defined by the four equations

$$
b_{i 1} y_{1}^{2}+b_{i 2} y_{2}^{2}+\ldots+b_{i 8} y_{8}^{2}=0 \quad, \quad 1 \leq i \leq 4 .
$$

In case $\mathfrak{A}$ is in general position, the space $Y$ is smooth (see Proposition 3.1.2 in [28]). There is a simple relation between the singular double octic $X$ and $Y$. To describe it, let $G_{1}=\mathbb{F}_{2}^{8}$ denote the elementary abelian 2-group of order 256. For $a=\left(a_{1}, \ldots, a_{8}\right) \in G_{1}$ we define an automorphism $\sigma_{a}: \mathbb{P}^{7} \rightarrow \mathbb{P}^{7}$ by

$$
\sigma_{a}\left(x_{1}: \cdots: x_{i}: \cdots: x_{8}\right)=\left((-1)^{a_{1}} x_{1}: \cdots:(-1)^{a_{i}} x_{i}: \cdots:(-1)^{a_{8}} x_{8}\right) .
$$

The group $G_{1}$ contains a distinguished normal subgroup $N_{1} \triangleleft G_{1}$ of index two, the kernel of the map $a \mapsto \sum_{i=1}^{8} a_{i}$.

Proposition 2.4. $X \cong Y / N_{1}$

Proof. First remark that the matrix $A$ defines a linear embedding $j: \mathbb{P}^{3} \rightarrow \mathbb{P}^{7}$ of projective spaces. As $\operatorname{Ker}(B)=\operatorname{Im}(A)$, the map $\pi_{1}: \mathbb{P}^{7} \rightarrow \mathbb{P}^{7},\left(y_{1}: \ldots: y_{8}\right) \mapsto$ $\left(y_{1}^{2}: \ldots: y_{8}^{2}\right)$ maps $Y$ onto the image of $j$ and realizes $\mathbb{P}^{3}$ as the quotient of $Y$ by $G_{1}$. The quotient map $\pi_{1}$ factors over $Y / N_{1}$, and the degree of $Y / N_{1}$ over $\mathbb{P}^{3}$ is two. The ramification locus of the resulting map $\alpha: Y / N_{1} \rightarrow \mathbb{P}^{3}$ is precisely $R$. Indeed, the nontrivial element in $G_{1} / N_{1}$ is represented by any vector $e_{i}=(0, \ldots, 1, \ldots, 0)$ 
in the standard bases of $\mathbb{F}_{2}^{8}, 1 \leq i \leq 8$. Consequently, the ramification locus consists of all points in $\mathbb{P}^{7}$ with one coordinate zero. The embedding $j: \mathbb{P}^{3} \rightarrow \mathbb{P}^{7}$ maps the planes $H_{1}, \ldots, H_{8}$ onto the intersection of $j\left(\mathbb{P}^{3}\right)$ with the coordinate hyperplanes in $\mathbb{P}^{7}$. As the double octic $X$ is uniquely determined by $R$, it follows $X=Y / N_{1}$.

This geometric relation between $Y, X$ and $\widetilde{X}$ immediately leads to the following isomorphism of Hodge-structures.

Proposition 2.5. For a given arrangement $\mathfrak{A}$ in general position there exists a natural isomorphism

$$
\mathrm{H}^{3}(\mathrm{Y}, \mathbb{Q})^{\mathrm{N}_{1}} \cong \mathrm{H}^{3}(\mathrm{X}, \mathbb{Q}) \cong \mathrm{H}^{3}(\widetilde{\mathrm{X}}, \mathbb{Q})
$$

of rational polarized Hodge structures. $\left(\right.$ Here $\mathrm{H}^{3}(\mathrm{Y}, \mathbb{Q})^{\mathrm{N}_{1}}$ denotes the subspace of invariants under $N_{1}$.)

Proof. As $X=Y / N_{1}$, one has immediately has $\mathrm{H}^{3}(\mathrm{Y}, \mathbb{Q})^{\mathrm{N}_{1}} \cong \mathrm{H}^{3}(\mathrm{X}, \mathbb{Q})$, showing that $H^{3}(X, \mathbb{Q})$ carries a pure Hodge structure. Let $\psi: \widetilde{X} \longrightarrow X$ be the resolution map described above. As in general the kernel of the map $\psi^{*}$ : $\mathrm{H}^{3}(X, \mathbb{Q}) \longrightarrow \mathrm{H}^{3}(\widetilde{X}, \mathbb{Q})$ is the part of smaller Hodge-weight (see Corollary 5.42 of [21]), we conclude that $\psi^{*}$ is injective. As the dimensions agree, $\phi^{*}$ is an isomorphism. (This, of course, can also directly be seen from the Leray spectral sequence for $\psi$.)

2.3. The hyperelliptic locus. There exist an interesting locus in $\mathfrak{M}_{A R}$ where the Hodge-structure is a third exterior power:

$$
\mathrm{H}^{3}(\widetilde{\mathrm{X}}, \mathbb{Q})=\wedge^{3} \mathrm{H}^{1}(\mathrm{C}, \mathbb{Q})
$$

where $C$ is a hyperelliptic curve of genus 3 . Such a $C$ is obtained by a two-fold cover of $\mathbb{P}^{1}$ ramified over eight points. There exists a natural morphism

$$
\gamma:\left(\mathbb{P}^{1}\right)^{3} \longrightarrow \mathbb{P}^{3}
$$

which sends the point $s=\left(\left(x_{1}: y_{1}\right),\left(x_{2}: y_{2}\right),\left(x_{3}: y_{3}\right)\right)$ to $\left(c_{0}(x, y): c_{1}(x, y)\right.$ : $\left.c_{2}(x, y): c_{3}(x, y)\right)$, where $x=\left(x_{1}, x_{2}, x_{3}\right), y=\left(y_{1}, y_{2}, y_{3}\right)$ and $c_{i}(x, y)$ is the $i$-th coefficient of the polynomial of the variables $t, s$

$$
\prod_{i=1}^{3}\left(x_{i} t+y_{i} s\right)=c_{0} s^{3}+c_{1} s^{2} t+c_{2} s t^{2}+c_{3} t^{3} \in \mathbb{C}[s, t] .
$$

This morphism $\gamma$ is Galois covering with Galois group $S^{3}$, the symmetric group acting on three letters.

One readily checks that for any $p=(a: b) \in \mathbb{P}^{1}$ the set $H:=\gamma\left(\{p\} \times \mathbb{P}^{1} \times \mathbb{P}^{1}\right)$ is the hyperplane with equation

$$
b^{3} z_{3}-a b^{2} z_{2}+a^{2} b z_{1}-a^{3} z_{0}=0 .
$$

We say that $H$ is the plane associated to the point $p$. 
Lemma 2.6. Let $\left(p_{1}, \ldots, p_{8}\right)$ denote a collection of eight distinct points in $\mathbb{P}^{1}$, and let $H_{i}:=\gamma\left(\left\{p_{i}\right\} \times \mathbb{P}^{1} \times \mathbb{P}^{1}\right)$ the associated planes. Then $\mathfrak{A}=\left(H_{1}, \ldots, H_{8}\right)$ is an arrangement of planes in general position.

Proof. In appropriate coordinates on $\mathbb{P}^{1}$, we may assume that $p_{i}=\left(-1: a_{i}\right)$ with $a_{i} \in \mathbb{C}$ pairwise distinct $(1 \leq i \leq 8)$. The matrix $A \in M(8 \times 4, \mathbb{C})$ corresponding to the arrangement $\mathfrak{A}$ then has the form

$$
A=\left(\begin{array}{cccc}
1 & a_{1} & a_{1}^{2} & a_{1}^{3} \\
1 & a_{2} & a_{2}^{2} & a_{2}^{3} \\
\vdots & \vdots & \vdots & \vdots \\
1 & a_{8} & a_{8}^{2} & a_{8}^{3}
\end{array}\right)
$$

Every $(4 \times 4)$-submatrix of $A$ is a Vandermonde matrix, hence its determinant is non-zero. This proves that $\mathfrak{A}$ is in general position.

Let $C$ be the hyperelliptic curve of genus 3 branched at $p_{1}, \ldots, p_{8} \in \mathbb{P}^{1}$, and let $q: C \rightarrow \mathbb{P}^{1}$ denote the corresponding covering map. The threefold product

$$
h: C^{3} \stackrel{q^{3}}{\longrightarrow}\left(\mathbb{P}^{1}\right)^{3} \stackrel{\gamma}{\longrightarrow} \mathbb{P}^{3}
$$

is a Galois covering of degree $2^{3} \cdot 6=48$. Its Galois group $G_{2}$ is isomorphic to a semi-direct product $N \rtimes S_{3}$, where $N=\left\langle\iota_{1}, \iota_{2}, \iota_{3}\right\rangle$ is the group generated by the hyperelliptic involution. We let $N_{2}$ denote the index two subgroup $N^{\prime} \rtimes S_{3}$ of $G_{2}$, where $N^{\prime}$ is the kernel of $N \cong \mathbb{F}_{2}^{3} \stackrel{\sum}{\longrightarrow} \mathbb{F}_{2}$, where the isomorphism sends $\iota_{1}$ to $(1,0,0)$ etc. We factor $h$ over the set of $N_{2}$-orbits and obtain a commutative diagram

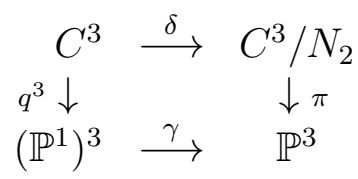

Lemma 2.7. The double cover $\pi: C^{3} / N_{2} \rightarrow \mathbb{P}^{3}$ branches along the union of the hyperplanes $H_{1}, \ldots, H_{8}$ associated to the points $p_{1}, \ldots, p_{8}$. So $C^{3} / N_{2} \cong X$, where $X$ is the double octic determined by $H_{1}, \ldots, H_{8}$.

Proof. The Galois group of $\pi$ is isomorphic to $G_{2} / N_{2}$, and it is generated by the image of $\iota_{1}$. Hence the ramification locus of $\pi$ is the image of the fixed locus $L_{1} \subseteq C^{3}$ of $\iota_{1}$ under $\pi \circ \delta$. By the commutativity of the above diagram, it coincides with the image of $L_{1}$ under $\gamma \circ q^{3}$. Obviously we have

$$
q^{3}\left(L_{1}\right)=\left\{p_{1}, \ldots, p_{8}\right\} \times \mathbb{P}^{1} \times \mathbb{P}^{1}
$$

so that our claim follows from Lemma 2.6. As $C^{3} / N_{2}$ and $X$ are both double covers of $\mathbb{P}^{3}$ with the same ramification divisor, they are isomorphic.

Proposition 2.8. Let $p_{1}, \ldots, p_{8}$ be eight distinct points in $\mathbb{P}^{1}, \mathfrak{A}$ the associated arrangement of hyperplanes and $\widetilde{X}$ the $C Y$ manifold which corresponds to this arrangement. Furthermore, let $q: C \rightarrow \mathbb{P}^{1}$ denote the hyperelliptic curve which ramifies at $p_{1}, \ldots, p_{8}$. Then we have an isomorphism of rational polarized Hodge structures

$$
\mathrm{H}^{3}(\widetilde{\mathrm{X}}, \mathbb{Q}) \cong \mathrm{H}^{3}(\mathrm{X}, \mathbb{Q}) \cong \wedge^{3} \mathrm{H}^{1}(\mathrm{C}, \mathbb{Q})
$$


Proof. As $X \cong C^{3} / N_{2}$, we have

$$
\mathrm{H}^{3}(\mathrm{X}, \mathbb{Q}) \cong \mathrm{H}^{3}\left(\mathrm{C}^{3}, \mathbb{Q}\right)^{\mathrm{N}_{2}} .
$$

Since $S_{3}$ is contained in $N_{2}$, we have an inclusion

$$
\mathrm{H}^{3}\left(\mathrm{C}^{3}, \mathbb{Q}\right)^{\mathrm{N}_{2}} \hookrightarrow \mathrm{H}^{3}\left(\mathrm{C}^{3}, \mathbb{Q}\right)^{\mathrm{S}_{3}} \cong \mathrm{H}^{3}\left(\operatorname{Sym}^{3}(\mathrm{C}), \mathbb{Q}\right) .
$$

Here $\operatorname{Sym}^{3}(C)$ denotes the symmetric threefold product of $C$, i.e. the quotient space $C^{3} / S_{3}$ where $S_{3}$ acts on $C^{3}$ by permutation of the factors. Let $J$ denote the three dimensional Jacobian of $C$. By the Abel-Jacobi theorem, the natural map $\varphi: \operatorname{Sym}^{3}(C) \rightarrow J$ is birational, and it induces an isomorphism between the middle cohomology spaces. Thus

$$
\mathrm{H}^{3}\left(\operatorname{Sym}^{3}(\mathrm{C}), \mathbb{Q}\right) \cong \mathrm{H}^{3}(\mathrm{~J}, \mathbb{Q}) \cong \wedge^{3} \mathrm{H}^{1}(\mathrm{~J}, \mathbb{Q}) \cong \wedge^{3} \mathrm{H}^{1}(\mathrm{C}, \mathbb{Q}) .
$$

Now the latter space is 20 dimensional, which is also the dimension of $H^{3}(\widetilde{\mathrm{X}}, \mathbb{Q})$. This shows that the inclusion $\mathrm{H}^{3}(\widetilde{\mathrm{X}}, \mathbb{Q}) \hookrightarrow \wedge^{3} \mathrm{H}^{1}(\mathrm{C}, \mathbb{Q})$ we constructed is actually an isomorphism.

Let $\mathfrak{M}_{8}$ denote the moduli space of eight points in $\mathbb{P}^{1}$, which is five dimensional. Lemma 2.6] shows that there exist a natural embedding $\mathfrak{M}_{8} \hookrightarrow \mathfrak{M}_{A R}$. We denote its image by $\widetilde{\mathfrak{H}}_{C Y}$ and its image in $\mathfrak{M}_{C Y}$ under the map $\mathfrak{M}_{A R} \rightarrow \mathfrak{M}_{C Y}$ by $\mathfrak{H}_{C Y}$. We call this the hyperelliptic sublocus.

Remark 2.9. It is worthwhile to remark that the construction generalizes to all $n \geq 2$. It produces a $2 n-1$ dimensional hyperelliptic locus in the $n^{2}$ dimensional moduli of CY manifolds, over which the primitive middle dimensional rational Hodge structures are wedge products of weight one Hodge structures. For $n=2$ the space $\mathfrak{M}_{C Y}$ arises from the moduli space of six lines in $\mathbb{P}^{2}$ in general position. In [17] the analogous sublocus $\mathfrak{H}_{C Y}$ was characterized as those six lines which are tangent to a smooth conic, and it was shown that it yields the family of Kummer surfaces.

\section{Classifying spaces and Canonical Variations}

We briefly recall some basic facts on Hodge structures and their classifying spaces. Let $V$ denote a real vector space, $n \in \mathbb{N}$ and $b: V \times V \rightarrow \mathbb{R}$ a non-degenerate bilinear form which is symmetric if $n$ is even and skew-symmetric if $n$ is odd. Furthermore, let $\left\{h^{p, q}\right\}$ denote a collection of non-negative integers parameterized by $(p, q) \in \mathbb{N}_{0}^{2}$ such that

$$
h^{p, q} \neq 0 \quad \text { only if } \quad p+q=n \quad \text { and } \quad h^{q, p}=h^{p, q} \quad \text { for all } \quad p, q \in \mathbb{Z} .
$$

The set $D$ of all real Hodge structures of type $\Phi=\left(V,\left\{h^{p, q}\right\}, b\right)$ is equipped with a natural structure of a complex manifold, called the classifying space of Hodge structures of type $\Phi$. It is a homogeneous space of the form $D=G / K$, where $G$ denotes the real Lie subgroup of $G L(V)$ consisting of all $\mathbb{R}$-linear automorphisms fixing $b$ and where $K$ denotes a compact subgroup of $G$. 
Now let $S$ be a complex manifold and fix a base point $s \in S$. Every $\mathbb{R}$-PVHS $\mathbb{V}$ of type $\Phi$ over $S$ gives rise to a map

$$
\phi: S \rightarrow \Gamma \backslash D
$$

called the period map associated to $\mathbb{V}$. Here $\Gamma$ is the image of the monodromy representation

$$
\tau: \pi_{1}(S, s) \rightarrow G
$$

defined by $\mathbb{V}$ considered as a local system of real vector spaces. We refer to Chapter I in [10] for more details.

In practice one often encounters situations where the real structure is lost. A typical example arises from the eigenspace decomposition of the complexification of the real cohomology of a cyclic cover with respect to the cyclic group action (see for example [7], [1]). Complex polarized variations of Hodge structures are also natural objects in Simpson's correspondence (see [23], §4). We will use this slightly generalized notion of $\mathbb{C}$-PVHS in this paper and we refer to $\S 1$ in [5] for the notions of complex Hodge structure $(\mathbb{C}-\mathrm{HS})$, complex polarized Hodge struc-

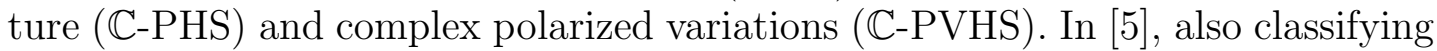
spaces of $\mathbb{C}$-PHS of with given Hodge numbers are defined. The corresponding classifying spaces of $\mathbb{C}$-PHS are also of form $D=G / K$ where $G$ is a real Lie group and $K$ is a compact subgroup of $G$. The difference from that of $\mathbb{R}$-PHS is in that $G$ is not necessarily $S p(2 n, \mathbb{R})$ or $S O(r, s)$, but can also be a special unitary group $S U(p, q)$.

\section{Locally homogenous and Canonical variations}

Let $D_{0}=G_{0} / K_{0}$ be a Hermitian symmetric domain (HSD) with $G_{0}$ the connected component of the automorphism group of $D_{0}$ and $K_{0}$ a maximal compact subgroup of $G_{0}$. There are four infinite series of classical domains after E. Cartan:

(I) $D_{p, q}^{I}=\frac{S U(p, q)}{S(U(p) \times U(q))}, p \geq q \geq 1$,

(II) $D_{n}^{I I}=\frac{S O^{*}(2 n)}{U(n)}, n \geq 5$,

(III) $D_{n}^{I I I}=\frac{S p(2 n, \mathbb{R})}{U(n)}, n \geq 2$,

(IV) $D_{n}^{I V}=\frac{S O_{0}(2, n)}{S O(2) \times S O(n)}, n \geq 5$.

Now let $\Gamma_{0}$ be a torsion free discrete subgroup of $G_{0}$. Let $\rho_{0}: G_{0} \rightarrow G L(F)$ be a finite dimensional complex representation of $G_{0}$. S. Zucker has shown in $\S 4$ of [33], that the complex local system $\mathbb{F}_{\Gamma_{0}}=\left(F \times_{\Gamma_{0}} D_{0}\right)$ over the complex manifold $\Gamma_{0} \backslash D_{0}$ admits naturally a structure of $\mathbb{C}$-PVHS, which we will call, following [33], a locally homogenous PVHS. By construction, it is clear that only when $\rho$ is defined over $\mathbb{R}, \mathbb{F}_{\Gamma_{0}}$ is a $\mathbb{R}$-PVHS.

The following example of locally homogenous PVHS was considered by B. Gross in [12] and also appeared in [27] in connection with moduli spaces of Calabi-Yau 
varieties. As explained in Proposition 1.2.6 in [5], $D_{0}$ determines a special node of the Dynkin diagram of the simple complex Lie algebra $\mathfrak{g}_{\mathbb{C}}=\operatorname{Lie}\left(G_{0}\right) \otimes \mathbb{C}$. By the standard theory on the finite dimensional representations of semi-simple complex Lie algebras, this node also determines a fundamental representation $W$ of $\mathfrak{g}_{\mathbb{C}}$, and $W$ integrates to an irreducible complex representation $\rho_{\text {can }}$ of $G_{0}$. When $D_{0}$ is of tube type, the representation $W$ is exactly the one considered by B. Gross in [12 and only in this case $W$ does admit an $G_{0}$-invariant real form. The locally homogenous PVHS by the above construction are of CY type (cf. $\S 1$ in [27]). Following Gross in [12, we call them canonical PVHS over $\Gamma_{0} \backslash D_{0}$. An important property of it is that the weight of $\mathbb{W}$ is equal to the rank of $D_{0}$.

Definition 3.1. Let $\left(D_{0}, \rho_{0}\right)$ be a pair consisting of a HSD $D_{0}$ and a homomorphism $\rho_{0}: G_{0} \rightarrow G$ of real Lie groups and let $\tilde{\psi}: D_{0} \rightarrow D$ the map induced from $\rho_{0}$ and

$$
\psi: \Gamma_{0} \backslash D_{0} \rightarrow \Gamma \backslash D, \quad \Gamma_{0}:=\rho_{0}^{-1}(\Gamma)
$$

1) We say that a $\mathbb{C}$-PVHS $\mathbb{V}$ over $S$ factors with respect to $\left(D_{0}, \rho_{0}\right)$, when the period map $\phi: S \rightarrow \Gamma \backslash D$ of $\mathbb{V}$ factors over $\psi$. That is, we have a diagram

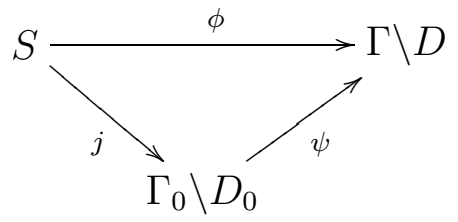

commutes.

2) For a $\mathbb{C}$-PVHS $\mathbb{V}$ of $\mathrm{CY}$ type we say that $\mathbb{V}$ factors canonically if it factors with respect to certain $\left(D_{0}, \rho_{\text {can }}\right)$

Lemma 3.2. If $\mathbb{V}$ factors with respect to $\left(D_{0}, \rho_{0}\right)$, then $j$ induces an isomorphism of $\mathbb{C}-P V H S \mathbb{V} \simeq j^{*} \mathbb{F}$, where $\mathbb{F}$ is the locally homogenous PVHS induced by $\rho_{0}$. Moreover the monodromy representation $\tau: \pi_{1}(S, s) \rightarrow G$ of $\mathbb{V}$ factorizes over $\rho_{0}$, i.e. there exists a homomorphism $\tau_{0}: \pi_{1}(S, s) \rightarrow G_{0}$ such that the diagram

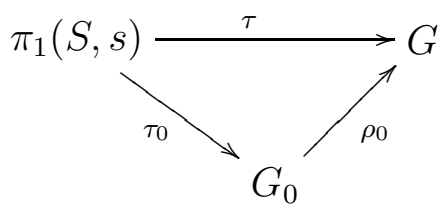

commutes.

Proposition 3.3. Let $\mathbb{V}$ be the $\mathbb{C}$-PVHS associated with a good family for the coarse moduli space $\mathfrak{M}_{C Y}$. If $\mathbb{V}$ factors canonically, then it must factor with respect to either $\left(D_{3,3}^{I}, \wedge^{3}\right)$ or $\left(D_{1,1}^{I} \times D_{8}^{I V}, i d \otimes i d\right)$.

Proof. Assume $\mathbb{V}$ factors with respect to $\left(D_{0}, \rho_{\text {can }}\right)$ for certain $\mathrm{HSD} D_{0}$. By the local Torelli theorem for Calabi-Yau manifolds we know that $D_{0}$ is at least 9 dimensional. We write $D_{0}=D_{1} \times \cdots \times D_{k}$ be the decomposition into product of irreducible HSD's. Then $\rho_{\text {can }}=\rho_{\text {can }, 1} \otimes \cdots \otimes \rho_{\text {can }, k}$ with $\rho_{\text {can }, i}$ canonical PVHS over $D_{i}$. By Schur's lemma $\mathbb{V} \simeq \mathbb{V}_{1} \otimes \cdots \otimes \mathbb{V}_{k}$ decomposes accordingly. Since the weight of $\mathbb{V}$ is 3 , then $k \leq 3$. 
Case $k=1$ : Then $D_{0}$ must be of tube type and $\mathbb{W}$ associated with $\rho_{\text {can }, 1}$ must be the canonical $\mathbb{R}$-PVHS. By the classification in [12], the pair in the statement is the only possibility with correct Hodge numbers.

Case $k=2$ : Since $D_{0}$ has rank 3 , we can assume $D_{1}$ has rank 1 and hence $D_{1}$ is the unit disk. It follows also the Hodge numbers of $\mathbb{V}_{1}$ over $D_{1}$ is 1,1 . Since $D_{2}$ supports canonical $\mathbb{R}$-PVHS, it must be a type IV domain. After checking the Hodge numbers, one sees immediately the pair $\left(D_{1,1}^{I} \times D_{8}^{I V}\right.$, id $\otimes$ id $)$ is the unique possibility.

Case $k=3$ :. It follows that each $D_{i}, i=1,2,3$ has rank 1 and so the dimension of $D_{1} \times D_{2} \times D_{3}$ is less than 9 .

\section{Characteristic Subvariety}

We refer the reader to $\S 3$ in [11] and references therein for an account of the theory of infinitesimal variations of Hodge structures, in short IVHS, initiated by P. Griffiths. There is an important series of invariants of IVHS of a $\mathbb{C}$-PVHS $\mathbb{V}$ of CY type over $S$, namely the characteristic subvarieties which are contained in the projectivized tangent bundle $\mathbb{P}\left(\mathcal{T}_{S}\right)$. The basic theory of characteristic subvarieties is developed in [27]. We recall the definition.

Definition 4.1. Let $\mathbb{V}$ a $\mathbb{C}$-PVHS of weight $n$ over $S$ of weight $n$ and $(E, \theta)$ the associated Higgs-bundle. For every $k$ with $1 \leq k \leq n$, the $k$-th iterated Higgs field defines a morphism

$$
\theta^{k}: \operatorname{Sym}^{k}\left(\mathcal{T}_{S}\right) \longrightarrow \operatorname{Hom}\left(E^{n, 0}, E^{n-k, k}\right)
$$

with dual map $\left(\theta^{k}\right)^{*}: \operatorname{Hom}\left(E^{n, 0}, E^{n-k, k}\right)^{*} \rightarrow \operatorname{Sym}^{k}\left(\Omega_{S}\right)$. Let $\mathfrak{a}_{k}$ denote the ideal generated by the image of $\left(\theta^{k}\right)^{*}$ in the symmetric algebra $\operatorname{Sym}^{\bullet}\left(\Omega_{S}\right)$. Then for every $k$ with $1 \leq k \leq n-1$, the projective variety

$$
\mathcal{C}_{k}=\operatorname{Proj}\left(\operatorname{Sym} \bullet\left(\Omega_{\mathrm{S}}^{1}\right) / \mathfrak{a}_{\mathrm{k}+1}\right) \subset \mathbb{P}\left(\mathcal{T}_{\mathrm{S}}\right)
$$

over $S$ is called the $k$-th characteristic subvariety of $\mathbb{V}$.

Remark 4.2. For a proper smooth family $f: \mathcal{X} \rightarrow S$ of CY $n$-folds the Yukawa coupling of $f$ is the section of $\operatorname{Sym}^{n}\left(\Omega_{S}^{1}\right) \otimes\left(R^{n} f_{*} \mathcal{O}_{\mathcal{X}}\right)^{\otimes 2}$ defined by the $n$-th iterated Kodaira-Spencer maps of $f$. It has significance in physics and is an important invariant in the study of geometry on moduli spaces of CY manifolds. Note that the $(n-1)^{t h}$ characteristic subvariety is just the vanishing locus of the Yukawa coupling.

Let $s \in S$ and $\left(\mathcal{C}_{k}\right)_{s}$ be the fiber of $\mathcal{C}_{k}$ over $s$, which is a subvariety of $\mathbb{P}\left(\mathcal{T}_{S, s}\right)$. following simple lemma characterizes the tangent vectors at $s$ whose classes lie in the reduced subvariety $\left(\mathcal{C}_{k}\right)_{s}^{\text {red }}$.

Lemma 4.3 (Lemma 3.2 in [27]). Let $v \in \mathcal{T}_{S, s}$ be a non-zero tangent vector, $[v]$ its class $\mathbb{P}\left(\mathcal{T}_{S, s}\right)$ and and $v^{k+1} \in \operatorname{Sym}^{k+1}\left(\mathcal{T}_{S, s}\right)$ the $k+1$-th symmetric tensor power of $v$. Then:

$$
[v] \in\left(\mathcal{C}_{k}\right)_{s}^{\text {red }} \subset \mathbb{P}\left(\mathcal{T}_{S, s}\right) \text { if and only if } v^{k+1} \in \operatorname{ker}\left(\theta^{k+1}\right) .
$$


Corollary 4.4. Let $\mathfrak{M}$ be a coarse moduli space of polarized smooth $C Y n$-folds and $f: \mathcal{X} \rightarrow S$ be a good family for it. Let $s \in S$ be a point in the ramification locus of the moduli map $S \rightarrow \mathfrak{M}$ of $f$. Then there is a projective linear subspace in $\left(\mathcal{C}_{k}\right)_{s}^{\text {red }}$ for $1 \leq k \leq n-1$.

Proof. The question is analytically local. Let $X$ be the fiber of $f$ over $s$ and $[s]$ be the image of $s$ in $\mathfrak{M}$. By the Bogomolov-Tian-Todorov theorem the differential of the moduli map of $f$ at $s$ is naturally identified with the Kodaira-Spencer map:

$$
\rho_{f, s}: \mathcal{T}_{S, s} \rightarrow \mathrm{H}^{1}\left(\mathrm{X}, \mathcal{T}_{\mathrm{X}}\right)
$$

Let $v \in \mathcal{T}_{S, s}$ be a nonzero tangent vector and $\omega$ be a generator of $\mathrm{H}^{0}\left(\mathrm{X}, \mathrm{K}_{\mathrm{X}}\right)$. By Griffiths we have the formula for the Higgs field action:

$$
\theta_{v}(\omega)=\rho_{f, s}(v) \cup \omega,
$$

where the cup product induces isomorphism $\mathrm{H}^{1}\left(\mathrm{X}, \mathcal{T}_{\mathrm{X}}\right) \otimes \mathrm{H}^{0}\left(\mathrm{X}, \mathrm{K}_{\mathrm{X}}\right) \simeq \mathrm{H}^{1}\left(\mathrm{X}, \Omega_{\mathrm{X}}^{\mathrm{n}-1}\right)$ for the CY manifold $X$. So it is clear that the kernel of $\rho_{f, s}$ is exactly the kernel of the Higgs field $\theta$ at $s$. In particular the corollary follows from Lemma 4.3 .

The main result in [27] identifies the characteristic subvarieties of the canonical PVHS over an irreducible HSD with the characteristic bundles introduced by $\mathrm{N}$. Mok in [15].

Theorem 4.5 (Theorem 3 in [27]). Let $D_{0}=G_{0} / K_{0}$ be an irreducible HSD of rank $n, \Gamma_{0}$ be a torsion free discrete subgroup of $G_{0}$ and let $(E, \theta)$ be the system of Hodge bundles associated to the canonical PVHS over $T:=\Gamma_{0} \backslash D_{0}$. Then for each $k$ with $1 \leq k \leq n-1$ the $k$-th characteristic subvariety $\mathcal{C}_{k}$ of $(E, \theta)$ over $T$ coincides with the $k$-th characteristic bundle $\mathcal{S}_{k}$ over $T$.

The characteristic bundles are first defined over $D_{0}$ and invariant under the $G_{0^{-}}$ action. By taking the quotient under the group $\Gamma$ one obtains the characteristic bundles on $T$. For each irreducible HSD the characteristic bundles are explicitly described in Appendix (III.3) in [16. For the purpose of this article we need only the information of the first characteristic bundle for the HSDs $D_{3,3}^{I}, D_{3}^{I I I}, D_{8}^{I V}$. For the convenience of the reader, we state them explicitly here. The following proposition is direct consequence of Theorem 4.5 .

Proposition 4.6. Notations as Theorem 4.5 and $t \in T$ arbitrary point. Then $\left(\mathcal{C}_{1}\right)_{t}$ as subvariety of $\mathbb{P}\left(\mathcal{T}_{T, t}\right)$ is isomorphic to

(i). $\mathbb{P}^{2} \times \mathbb{P}^{2} \hookrightarrow \mathbb{P}^{8}$ with the Segre embedding, when $D_{0}=D_{3,3}^{I}$,

(ii). $\mathbb{P}^{2} \hookrightarrow \mathbb{P}^{5}$ with the Veronese embedding, when $D_{0}=D_{3}^{I I I}$,

(iii). $Q \hookrightarrow \mathbb{P}^{7}$ with $Q$ a smooth quadratic hypersurface, when $D_{0}=D_{8}^{I V}$.

For the application we also need to work out the first characteristic subvariety in the reducible case $D_{0}=D_{1,1}^{I} \times D_{8}^{I V}$.

Proposition 4.7. Notations as last proposition. Let $D_{0}$ be the $H S D D_{1,1}^{I} \times$ $D_{8}^{I V}$. Then for each point of $t \in T,\left(\mathcal{C}_{1}\right)_{t}$ as subvariety of $\mathbb{P}\left(\mathcal{T}_{T, t}\right)$ is isomorphic to a disjoint union of a point $P$ with a smooth quadratic hypersurface $Q$ in a hyperplane of $\mathbb{P}^{8}$ away from $P$. In particular, it is not equidimensional. 
Proof. We put $D_{1}=D_{1,1}^{I}$ and $D_{2}=D_{8}^{I V}$ and fix the base point $0 \in D_{0}$. Then the canonical PVHS $\mathbb{W}$ over $D_{0}$ is given by tensor product $\mathbb{W}_{1} \otimes \mathbb{W}_{2}$ of the canonical PVHS $\mathbb{W}_{i}$ over $D_{i}, i=1,2$. Let $\left(E_{i}, \theta_{i}\right)$ be the corresponding Higgs-bundle to $\mathbb{W}_{i}$. Then the corresponding Higgs-bundle $(E, \theta)$ to $\mathbb{W}$ is given by $\left(E_{1} \otimes E_{2}, \theta_{1} \otimes\right.$ $\left.i d+i d \otimes \theta_{2}\right)$. Let $p_{i}: D_{0} \rightarrow D_{i}, i=1,2$ be the natural projection. Then one has natural isomorphism $\mathcal{T}_{D_{0}} \simeq p_{1}^{*}\left(\mathcal{T}_{D_{1}}\right) \oplus p_{2}^{*}\left(\mathcal{T}_{D_{2}}\right)$. Under this isomorphism, we represent a tangent vector $v \in \mathcal{T}_{D_{0}, 0}$ by a pair $\left(v_{1}, v_{2}\right)$ with $v_{i} \in \mathcal{T}_{D_{i}, 0}$. Take a nonzero vector $e=e_{1} \otimes e_{2} \in\left(E_{1}^{1,0} \otimes E_{2}^{2,0}\right)_{0}$. Then by Lemma 4.3, the fiber over 0 of the first characteristic subvariety of $D_{0}$ is determined by

$$
\left\{[v] \in \mathbb{P}\left(\mathcal{T}_{D_{0}, 0}\right) \mid\left(\theta_{v}\right)^{2}(e)=0\right\} .
$$

A simple calculation shows that

$$
\left(\theta_{v}\right)^{2}(e)=2\left(\left(\theta_{1}\right)_{v_{1}}\left(e_{1}\right) \otimes\left(\theta_{2}\right)_{v_{2}}\left(e_{2}\right)\right)+e_{1} \otimes\left(\left(\theta_{2}\right)_{v_{2}}\right)^{2}\left(e_{2}\right) .
$$

Hence $v$ is a characteristic vector if and only if $v_{2}=0$ or, $v_{1}=0$ and $v_{2}$ is a characteristic vector in $\mathcal{T}_{D_{2}, 0}$. Thus the fiber over 0 of the first characteristic subvariety is the disjoint union of a point with the fiber over 0 of the first characteristic subvariety of $D_{2}$, which is isomorphic to a smooth quadratic hypersurface in $\mathbb{P}^{7}$ by Proposition 4.6 (iii).

Corollary 4.8. If $\mathbb{V}$ factors canonically, then for any $s \in S$ away from the ramification locus of the moduli map $S \rightarrow \mathfrak{M}_{C Y}$, then $\left(\mathcal{C}_{1}\right)_{s} \subset \mathbb{P}\left(\mathcal{T}_{S, s}\right)$ is isomorphic to $\mathbb{P}^{2} \times \mathbb{P}^{2} \subset \mathbb{P}^{8}$ or the $P \cup Q \subset \mathbb{P}^{8}$ as in Proposition 4.7 .

Proof. This is a consequence of Proposition 3.3 and Propositions 4.6, 4.7,

\section{Explicit Infinitesimal Variation of Hodge Structures of the Calabi-Yau Threefolds}

In those cases where there is an explicit description of the cohomology with the help of an jacobian ring, it is usually also possible to construct the infinitesimal invariants of the corresponding variation. In particular, it will be possible to compute the characteristic subvarieties. We will illustrate this for the local system $\mathbb{V}$ for a good family of double octics $f: \mathcal{X} \rightarrow S$ with fibres $\widetilde{X}$ covered by $Y$.

5.1. Jacobian Rings. As $Y$ is a complete intersection, one can find a description of the cohomology in terms of a certain Jacobian ring with the aid of the Cayleytrick. Let

$$
R_{8,4}=\mathbb{C}\left[x_{1}, \ldots, x_{8}, y_{1}, \ldots, y_{4}\right]
$$

denote the polynomial ring over $\mathbb{C}$ in 12 variables. Let the four quadrics defining $Y$ are given by

$$
f_{i}=\sum_{j=1}^{8} b_{i j} x_{j}^{2}
$$

and define

$$
F=\sum_{i=1}^{4} y_{i} f_{i} \in R_{8,4}
$$


Let $\mathfrak{J}_{\mathfrak{A}}$ denote the homogenous ideal generated by the twelve partial derivatives $\frac{\partial F}{\partial x_{j}}$ and $\frac{\partial F}{\partial y_{i}}$, and put

$$
R_{Y}=R_{8,4} / \mathfrak{J}_{\mathfrak{A}} .
$$

There is a natural bigrading on $R_{8,4}$ which assigns the value $(0,1)$ to each of the variables $x_{i}$ and $(1,-2)$ to each $y_{i}$. It induces a bigrading on $R_{Y}$. For $0 \leq p \leq 3$ we let $R_{Y}^{(p)}$ denote the subspace generated by monomials of bidegree $(p, 0)$, that is, monomials whose total degree in the $x_{j}$ is $2 p$ and whose total degree in the $y_{i}$ is $p$.

Proposition 5.1. There is an isomorphism

$$
\mathrm{H}^{3}(\mathrm{Y}, \mathbb{C}) \cong \mathrm{R}_{\mathrm{Y}}
$$

of $\mathbb{C}$-vector spaces which identifies

$$
\mathrm{H}^{3-\mathrm{p}, \mathrm{p}}(\mathrm{Y}) \cong \mathrm{R}_{\mathrm{Y}}^{(\mathrm{p})}, \quad 0 \leq \mathrm{p} \leq 3
$$

Proof. See [19], Prop. 2.2.10 on page 40 or [29].

In $\$ 2.2$ we defined the group $G_{1}$ of sign-changes and its subgroup $N_{1}$ of index two. These groups naturally act on the polynomial ring $R_{8,4}$ by sign-changes on the $x_{j}, 1 \leq j \leq 8$ and trivially on the $y_{i}, 1 \leq i \leq 4$. There is an induced action of $N_{1}$ on the quotient $R_{Y}$. We let $\widetilde{R}_{Y}$ denote the subring $R_{Y}^{N_{1}}$ of elements in $R_{Y}$ fixed by every $\sigma \in N_{1}$.

Corollary 5.2. It induces an isomorphism between the subspaces

$$
\mathrm{H}^{3}(\widetilde{\mathrm{X}}, \mathbb{C}) \cong \widetilde{\mathrm{R}}_{\mathrm{Y}}
$$

which is also compatible with the Hodge decomposition and the total grading.

Proof. By Proposition 3.4, $\mathrm{H}^{3}(\widetilde{\mathrm{X}}, \mathbb{Q})$ is the subspace of $\mathrm{H}^{3}(\mathrm{Y}, \mathbb{Q})$ invariant under $N_{1}$-action and moreover is a sub PHS of $\mathrm{H}^{3}(\mathrm{Y}, \mathbb{Q})$. So one has

$$
\mathrm{F}^{\mathrm{p}} \mathrm{H}^{3}(\widetilde{\mathrm{X}}, \mathbb{C})=\mathrm{F}^{\mathrm{p}} \mathrm{H}^{3}(\mathrm{Y}, \mathbb{C}) \cap \mathrm{H}^{3}(\widetilde{\mathrm{X}}, \mathbb{C})=\left(\mathrm{F}^{\mathrm{p}} \mathrm{H}^{3}(\mathrm{Y}, \mathbb{C})\right)^{\mathrm{N}_{1}}
$$

So the assertion follows.

\subsection{Multiplication and Cup-Product.}

Proposition 5.3. For $0 \leq p \leq 2$ the following diagram commutes

$$
\begin{array}{ccc}
\mathcal{T}_{S, s} \otimes \mathrm{H}^{3-\mathrm{p}, \mathrm{p}}(\widetilde{\mathrm{X}}) & \stackrel{\theta_{s}}{\longrightarrow} & \mathrm{H}^{2-\mathrm{p}, \mathrm{p}+1}(\widetilde{\mathrm{X}}) \\
\cong \downarrow & \downarrow \cong \\
\widetilde{R}_{Y}^{(1)} \otimes \widetilde{R}_{Y}^{(p)} & \stackrel{\mu}{\longrightarrow} & \widetilde{R}_{Y}^{(p+1)} .
\end{array}
$$

Here the vertical arrows are provided by Proposition 5.1 and the above isomorphism, and the lower horizontal arrow is the ring multiplication map.

Proof. First we recall that in $\S 3$ we associate each element in $S$ a smooth complete intersection of four quadrics $Y$ in $\mathbb{P}^{7}$. Let $g_{0}: \mathcal{Z} \rightarrow Z$ be a good family over the 
moduli space of smooth complete intersection of four quadrics in $\mathbb{P}^{7}$. So one has the natural closed embedding $S \hookrightarrow Z$. Put

$$
h_{0}=\left.g_{0}\right|_{S}: \mathcal{Y}=\left.\mathcal{Z}\right|_{g_{0}^{-1}(S)} \rightarrow S .
$$

Globalizing the construction in Proposition 2.5 to $h_{0}$, one obtains a new family $\tilde{h}_{0}: \widetilde{\mathcal{Y}} \rightarrow S$ admitting $N_{1}$-action over $S$, and by taking quotient of it under $N_{1^{-}}$ action one recovers the family $f_{0}: \mathcal{X} \rightarrow S$. In summary, one has the following commutative diagrams

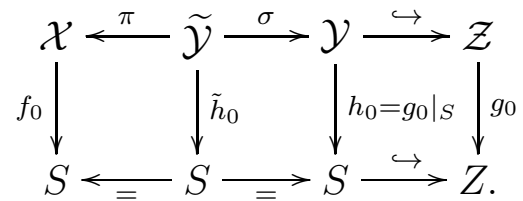

Now we let $\mathbb{W}=R^{3} g_{0 *} \mathbb{Q}_{\mathcal{Z}}$ and $(F, \eta)$ be the system of Hodge bundles associated with $\mathbb{W}$. By construction, it is clear that $\mathbb{V} \simeq\left(\left.\mathbb{W}\right|_{S}\right)^{N_{1}}$ as PVHS. It follows that for $v \in \mathcal{T}_{S, s} \subset \mathcal{T}_{Z, s}$ one has natural identification

$$
\theta_{s}(v)(\alpha)=\eta_{s}(v)(\alpha)
$$

where $\alpha \in \mathrm{H}^{3-\mathrm{p}, \mathrm{p}}(\widetilde{\mathrm{X}}) \simeq \mathrm{H}^{3-\mathrm{p}, \mathrm{p}}(\mathrm{Y})^{\mathrm{N}_{1}} \subset \mathrm{H}^{3-\mathrm{p}, \mathrm{p}}(\mathrm{Y})$.

Furthermore, for the IVHS of $\mathbb{W}$ at $s$, one has the following commutative diagram (see Proposition 2.6 in [29])

$$
\begin{array}{cccc}
\mathcal{T}_{Z, s} & \stackrel{\eta_{s}}{\longrightarrow} & \bigoplus_{p} \operatorname{Hom}\left(\mathrm{H}^{3-\mathrm{p}, \mathrm{p}}(\mathrm{Y}), \mathrm{H}^{2-\mathrm{p}, \mathrm{p}+1}(\mathrm{Y})\right) \\
& \downarrow \downarrow & & \\
R_{Y}^{(1)} & \longrightarrow & \bigoplus_{p} \operatorname{Hom}\left(R_{Y}^{(p)}, R_{Y}^{(p+1)}\right),
\end{array}
$$

where the lower horizontal arrow is induced by the ring multiplication map.

Finally one has the following commutative diagrams by the construction of the families $h_{0}, \tilde{h}_{0}$ and $f_{0}$ :

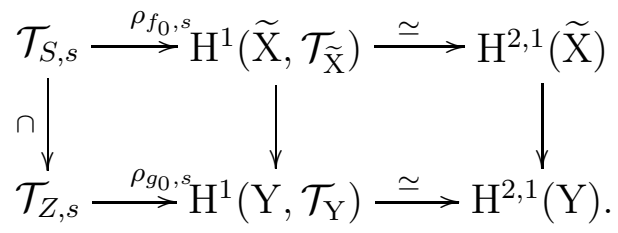

This shows that under the left vertical isomorphism $\mathcal{T}_{Z, s} \rightarrow R_{Y}^{(1)}$ (in the second paragraph), the image of $\mathcal{T}_{S, s}$ is exactly $\widetilde{R}_{Y}^{(1)}$. The proposition follows by putting everything above together.

5.3. Calculation. Now we proceed to describe the computation of the characteristic subvarieties introduced in \$4. According to Proposition 5.3, the action of Higgs field on the cohomology classes along a given tangent vector is equivalent to the multiplication of corresponding elements in $\widetilde{R}_{Y}$ with some fixed element 
in $\widetilde{R}_{Y}^{(1)}$. Furthermore, since $\widetilde{R}_{Y}^{(0)}$ is one-dimensional, there exists a isomorphism $\operatorname{Hom}\left(\widetilde{R}_{Y}^{(0)}, \widetilde{R}_{Y}^{(k)}\right) \cong \widetilde{R}_{Y}^{(k)}$. Hence in our case the $k$-th iterated Higgs fields

$$
\left(\theta_{s}\right)^{k}: \operatorname{Sym}^{k}\left(T_{S, s}\right) \longrightarrow \operatorname{Hom}\left(\mathrm{H}^{3,0}(\widetilde{\mathrm{X}}), \mathrm{H}^{3-\mathrm{k}, \mathrm{k}}(\widetilde{\mathrm{X}})\right)
$$

is given by the multiplication map

$$
\mu_{k}: \operatorname{Sym}^{k}\left(\widetilde{R}_{Y}^{(1)}\right) \longrightarrow \widetilde{R}_{Y}^{(k)} .
$$

It follows that the $k$-th characteristic subvariety at $s$ is isomorphic to

$$
\left(\mathcal{C}_{k}\right)_{s}=\mathbb{P}\left(\operatorname{Sym} \cdot\left(\left(\widetilde{R}_{Y}^{(1)}\right)^{*} / \mathfrak{a}_{k+1}\right),\right.
$$

where $\mathfrak{a}_{k+1}$ denotes the image of the dual $\mu_{k+1}^{*}:\left(\widetilde{R}_{Y}^{(k+1)}\right)^{*} \rightarrow \operatorname{Sym}^{k+1}\left(\left(\widetilde{R}_{Y}^{(1)}\right)^{*}\right)$ of the multiplication map. Fixing a basis of $\widetilde{R}_{Y}^{(1)}$ determines an isomorphism $\mathbb{P}\left(\operatorname{Sym} \cdot\left(\left(\widetilde{R}_{Y}^{(1)}\right)^{*}\right)\right) \cong \mathbb{P}^{8}$. This shows that the first characteristic subvariety $\left(\mathcal{C}_{1}\right)_{s}$ can be computed by the following steps. Let $\left(u_{1}, \ldots, u_{9}\right)$ and $\left(v_{1}, \ldots, v_{9}\right)$ denote the elements of $\mathcal{B}_{1}$ and $\mathcal{B}_{2}$, respectively.

(1) Fix a bijection

$$
\phi:\left\{(i, j) \in \mathbb{N}^{2} \mid 1 \leq i \leq j \leq 9\right\} \stackrel{\sim}{\longrightarrow}\{1, \ldots, 45\} \quad,
$$

and define a basis $\mathcal{B}=\left(w_{1}, \ldots, w_{45}\right)$ of $\operatorname{Sym}^{2}\left(\widetilde{R}_{Y}^{(1)}\right)$ by $w_{\phi(i, j)}=u_{i} u_{j}$. Compute the representation matrix $C \in M(9 \times 45, \mathbb{C})$ of the multiplication map $\mu_{2}$ with respect to $\mathcal{B}$ and $\mathcal{B}_{2}$.

(2) Let $\mathcal{B}_{1}^{*}=\left(u_{1}^{*}, \ldots, u_{9}^{*}\right)$ denote the dual basis of $\mathcal{B}_{1}$, and let the basis $\widetilde{\mathcal{B}}=$ $\left(\tilde{w}_{1}, \ldots, \tilde{w}_{45}\right)$ of $\operatorname{Sym}^{2}\left(\left(\widetilde{R}_{Y}^{(1)}\right)^{*}\right)$ be defined by $\tilde{w}_{\phi(i, j)}=u_{i}^{*} u_{j}^{*}$. Determine the representation matrix $D=\left(d_{i j}\right) \in M(45 \times 9, \mathbb{C})$ of the dualized multiplication $\mu_{2}^{*}$ with respect to $\mathcal{B}_{2}^{*}$ and $\widetilde{\mathcal{B}}$.

(3) For the $k$-th column of $D$ define a polynomial $f_{k} \in \mathbb{C}\left[z_{1}, \ldots, z_{9}\right]$ by

$$
f_{k}=\sum_{i=1}^{9} \sum_{j=i}^{9} d_{\phi(i, j) k} z_{i} z_{j} .
$$

Let $\mathfrak{a}_{2}$ be the ideal generated by $f_{1}, \ldots, f_{9}$. Then $\left(\mathcal{C}_{1}\right)_{s}$ is isomorphic to the projective subvariety in $\mathbb{P}^{8}$ which corresponds to $\mathfrak{a}_{2}$.

Remark 5.4. Step (1) can be carried out in practice using computer algebra. For each pair $(i, j)$, one computes the product $g_{1} g_{2}$ of the polynomials $g_{i}, g_{j} \in R_{8,4}$ which correspond to the basis vectors $w_{i}, w_{j} \in \mathcal{B}_{1}$ and reduces it with respect to the Jacobian ideal $\mathfrak{J}_{\mathfrak{A}}$. The result can be expressed as a linear combination of the elements in $\mathcal{B}_{2}$. For step (2) notice that $\widetilde{\mathcal{B}}$ is related to the dual basis $\widetilde{\mathcal{B}}^{*}$ of $\mathcal{B}$ by

$$
\tilde{w}_{\phi(i, j)}=\left\{\begin{array}{lll}
\tilde{w}_{\phi(i, j)}^{*} & \text { if } \quad i=j \\
\frac{1}{2} \tilde{w}_{\phi(i, j)}^{*} & \text { if } \quad i \neq j .
\end{array}\right.
$$

By a similar procedure, we can compute the characteristic subvariety $\left(\mathcal{C}_{2}\right)_{s}$. We skip the details here, because it will not be used in the sequel. 
5.4. A general point and a special point. Here we summarize the results of the calculations that can be performed by the method explained above. First, if we pick a generic configuration $\mathfrak{A}$ giving a general point $\eta_{0} \in \mathfrak{M}_{C Y}$ and determine the ideal

$$
\mathfrak{a}_{2} \subset \mathbb{C}\left[z_{1}, \ldots, z_{9}\right]
$$

consisting of 9 quadratic polynomials. Using a Gröbner-basis calculation one can verify the following (see [9] for details):

Proposition 5.5. The characteristic subvariety $\left(\mathcal{C}_{1}\right)_{\eta_{0}}$ over a general point $\eta_{0}$ in the moduli space $\mathfrak{M}_{C Y}$ is empty set.

We will now chooses a special point $s_{0} \in \widetilde{\mathfrak{H}}_{C Y}$ represented by the matrix $A \in$ $M(8 \times 4, \mathbb{C})$ with

$$
A^{t}=\left(\begin{array}{cccccccc}
1 & 1 & 1 & 1 & 1 & 1 & 1 & 1 \\
1 & 2 & 3 & 4 & 5 & 6 & 7 & 8 \\
1 & 4 & 9 & 16 & 25 & 36 & 49 & 64 \\
1 & 8 & 27 & 64 & 125 & 216 & 343 & 512
\end{array}\right)
$$

Proposition 5.6. The characteristic subvariety $\left(\mathcal{C}_{1}\right)_{s_{0}}^{\text {red }}$ consists of two irreducible surfaces of degree 6 , each spanning the same $\mathbb{P}^{7} \subset \mathbb{P}^{8}$.

Remark 5.7. For the point $s_{0}$ the ideal $\mathfrak{a}_{2} 9$ quadratic polynomials in the variables $z_{1}, z_{2}, \ldots, z_{9}$. We analyzed this ideal using the computer algebra system Singular. Via a Hilbert-series computation, one shows that the variety in $\mathbb{P}^{8}$ has dimension two an is of degree 12. In order to find the irreducible components of this surface, one has to make a primary decomposition of the ideal $\mathfrak{a}_{2}$. For this we had to use several tricks.

By elimination of the first five variables we obtain an hypersurface of degree 8 in variables $z_{6}, z_{7}, z_{8}, z_{9}$. By looking at reductions modulo various primes $p$, it was observed that the octic factors as product of two quartics over $\mathbb{F}_{p}$ in case $p=1 \bmod 4$. With some more work one finds a factorization of the octic into two quartics over the field $\mathbb{Q}(\sqrt{-1})$.

Both quartics have a smooth twisted cubic as singular locus. Because the generic plane section is a three nodal quartic, we see that these two quartic surfaces are irreducible over $\mathbb{C}$. The decomposition of the degree 8 surface into two quartics gives a splitting of the degree 12 surface in $\mathbb{P}^{8}$ into two components, which are surfaces of degree 6 .

The change in degree from 12 to 8 is due to the fact the projecting out the first five variables is non-generic. If instead we eliminate the variables $z_{1}, z_{5}, z_{6}, z_{8}, z_{9}$ one finds that the degree 6 components project to sextic surfaces, whose plane section is a 10-nodal sextic, hence rational. Such surfaces are in fact ruled and can be obtained as join in $\mathbb{P}^{7}$ of corresponding points on a conic and rational normal curve of degree four and are cut out by 15 quadrics in $\mathbb{P}^{7}$. Indeed, it turns 
out that both components are contained in the hyperplane of $\mathbb{P}^{8}$ given by

$$
2269 z_{1}-378 z_{2}+21 x_{3}-1029 z_{4}+147 z_{5}-7 z_{6}+192 z_{7}-24 z_{8}+z_{9}=0
$$

By taking ideal quotients one can find a complete primary decomposition of the ideal $\mathfrak{a}_{2}$. There are embedded components of dimension one contained in the union of the two scrolls.

\section{Proof of the Main Theorems}

Theorem 6.1. Let $f: \mathcal{X} \rightarrow S$ be a good family of $\mathfrak{M}_{C Y}$ and $\mathbb{V}$ be the associated weight 3 PVHS. Then $\mathbb{V}$ does not factor canonically.

Proof. Assume the contrary. By Corollary 4.8, for any $s \in S$ away from the ramification locus of the moduli map $S \rightarrow \mathfrak{M}_{C Y}$ the first characteristic subvariety $\left(\mathcal{C}_{1}\right)_{s}$ is then isomorphic to either $\mathbb{P}^{2} \times \mathbb{P}^{2}$ or $P \cup Q$. In particular, in both cases there exists an irreducible component in $\left(\mathcal{C}_{1}\right)_{s}$ whose dimension is greater than 2. By Proposition 5.5 the fiber of the first characteristic subvariety at a general point $\left[\eta_{0}\right]$, the image of $\eta_{0}$ in $\mathfrak{M}_{C Y}$, is empty. By semi-continuity, there is an open neighborhood of $\left[\eta_{0}\right]$ in $\mathfrak{M}_{C Y}$ such that the fibers of the first characteristic subvariety over the closed points in it are empty. Therefore there exists also a closed point in $S$ away from the ramification locus of the moduli map of $f$, over which the fiber of the first characteristic subvariety is empty. This gives a contradiction.

Our next aim is to prove Theorem 6.3. For the proof of it, we use the notations as in $\S 1$. We first prove the following

Lemma 6.2. Each factor $\mathbb{V}_{i}$ has quasi-unipotent local monodromy around each irreducible component of $Z$.

Proof. We put $\mathbb{V}^{\prime}=\mathbb{V}_{2} \otimes \cdots \otimes \mathbb{V}_{k}$ and $n=\operatorname{rank} \mathbb{V}^{\prime}$. One considers the $\mathbb{C}$-PVHS $\bigwedge^{n} \mathbb{V}=\bigwedge^{n}\left(\mathbb{V}_{1} \otimes \mathbb{V}^{\prime}\right)$. By Exercise 6.11 (b) in [8] $\operatorname{Sym}^{n} \mathbb{V}_{1}$ is a direct factor of $\Lambda^{n} \mathbb{V}$. Since $\mathbb{V}$ is of quasi-unipotent local monodromy, the same holds for each direct factor of $\bigwedge^{n} \mathbb{V}$, in particular for $\operatorname{Sym}^{n}\left(\mathbb{V}_{1}\right)$. Thus one induces that $\mathbb{V}_{1}$ itself is also of quasi-unipotent local monodromy, and therefore so is $\mathbb{V}^{\prime}$. By induction on the number of factors $k$ in the tensor decomposition of $\mathbb{V}$, one concludes that each factor has quasi-unipotent local monodromy.

Theorem 6.3. Each local system $\mathbb{V}_{i}$ admits the structure of a $\mathbb{C}$-PVHS such that the induced $\mathbb{C}$-PVHS on the tensor product $\mathbb{V}_{1} \otimes \cdots \otimes \mathbb{V}_{k}$ coincides with the given $\mathbb{C}$-PVHS on $\mathbb{V}$.

Proof. Let $s \in S$ be a base point and let $\rho_{i}: \pi_{1}(S, s) \rightarrow G_{i}$ be the monodromy representation of $\mathbb{V}_{i}$. We put $V_{1}$ to be the fiber of $\mathbb{V}_{1}$ at $s$. Since $\rho_{i}$ is a Zariski dense representation into the simple Lie group $G_{i}$ with quasi-unipotent local monodromy around $Z$, by [13] there exists a pluri-harmonic metric on the flat bundle $\mathbb{V}_{i}$ with finite energy, which makes $\mathbb{V}_{i}$ into a Higgs bundle $\left(E_{i}, \theta_{i}\right)$ over $S$. Furthermore in [14] Mochizuki has analyzed the singularity of this harmonic 
metric in detail and has shown that $\left(E_{i}, \theta_{i}\right)$ admits a logarithmic extension $\left(\bar{E}_{i}, \bar{\theta}_{i}\right)$ over $\bar{S}$, i.e. a vector bundle $\bar{E}_{i}$ over $\bar{S}$ which extends $E_{i}$ and a map

$$
\bar{\theta}: \bar{E}_{i} \longrightarrow \bar{E}_{i} \otimes \Omega_{\bar{S}}^{1}(\log Z)
$$

which coincides with $\theta$ over $S$. Such a pluri-harmonic metric is called tame. In this case the residue of $\bar{\theta}$ along $Z$ is unipotent.

From the proof of Lemma 6.2 we know that one finds a non-trivial component $\operatorname{Sym}^{n}\left(\mathbb{V}_{1}\right)$ in $\bigwedge^{n}\left(\mathbb{V}_{1} \otimes \mathbb{V}^{\prime}\right)$. Since $\rho_{1}$ is Zariski dense in $G_{1}$ and $G_{1}$ is simple, one finds a suitable Schur functor $\mathbb{S}_{\mu}$ such that $\mathbb{S}_{\mu}\left(\rho_{1}\right)$ is a nontrivial irreducible direct factor of $\bigwedge^{n}\left(\mathbb{V}_{1} \otimes \mathbb{V}^{\prime}\right)$. Since $\Lambda^{n}\left(\mathbb{V}_{1} \otimes \mathbb{V}^{\prime}\right)$ is semi-simple, there exists a decomposition

$$
\bigwedge^{n}\left(\mathbb{V}_{1} \otimes \mathbb{V}^{\prime}\right)=\bigoplus_{i=1}^{m} \mathbb{W}_{i 1} \otimes \mathbb{W}_{i 2}
$$

where the $\mathbb{W}_{i 1}$ are irreducible and the $\mathbb{W}_{i 2}$ are trivial. By Proposition 1.13 in [5], there exist uniquely determined $\mathbb{C}$-PVHS on the $\mathbb{W}_{i 1}$ and complex Hodge structures on the $\mathbb{W}_{i 2}$ such that the direct sum of the tensor products of them coincides with the $\mathbb{C}$-PVHS on $\bigwedge^{n}\left(\mathbb{V}_{1} \otimes \mathbb{V}^{\prime}\right)$. In particular, there exists a $\mathbb{C}$-PVHS on $\mathbb{S}_{\mu}\left(\mathbb{V}_{1}\right)$.

By the uniqueness of the such pluri-harmonic metrics, the induced pluri-harmonic metric on $\mathbb{S}_{\mu}\left(\bar{E}_{1}, \bar{\theta}_{1}\right)$ coincides with that of the $\mathbb{C}$-PVHS on $\mathbb{S}_{\mu}\left(\mathbb{V}_{1}\right)$. Hence $\mathbb{S}_{\mu}\left(\bar{E}_{1}, \bar{\theta}_{1}\right)$ is a fixed point of the $\mathbb{C}^{\times}$-action. The Schur functor $\mathbb{S}_{\mu}$ induces a nontrivial morphism $G_{1} \rightarrow G L\left(\mathbb{S}_{\mu}\left(V_{1}\right)\right)$, which is injective since $G_{1}$ is simple. It induces the morphism

$$
\phi_{\mu}: \mathfrak{M}\left(\pi_{1}(S), G_{1}\right)^{\mathrm{ss}} \longrightarrow \mathfrak{M}\left(\pi_{1}(S), \mathrm{GL}\left(\mathbb{S}_{\mu}\left(V_{1}\right)\right)\right)^{\mathrm{ss}}
$$

between the corresponding moduli spaces of semi-simple representations. By Corollary 9.16 in [24], the morphism $\phi_{\mu}$ is finite.

If $Z=\emptyset$, then $\mathbb{C}^{\times}$acts on both moduli spaces continuously via the Hermitian Yang-Mills metric on poly-stable Higgs bundles $(E, t \theta)$, and this action is compatible with $\phi_{\mu}$. Since $\mathbb{S}_{\mu}\left(\rho_{1}\right)$ is a fixed point of the $\mathbb{C}^{\times}$-action, the representation $\rho_{1}$ itself is a fixed point of the $\mathbb{C}^{\times}$-action. Hence $\left(E_{1}, \theta_{1}\right)$ is a $\mathbb{C}$-PVHS on $\mathbb{V}_{1}$. Now we consider the general situation $Z \neq \emptyset$. Let $C \subseteq \bar{S}$ denote a curve which is a complete intersection of ample hypersurfaces, and define $C_{0}=C \backslash Z$. Taking the restriction

$$
\left.\rho_{1}\right|_{C_{0}} \in \mathfrak{M}\left(\pi_{1}\left(C_{0}\right), G_{1}\right)^{\mathrm{ss}},
$$

we have $\left.\mathbb{S}_{\mu}\left(\rho_{1}\right)\right|_{C_{0}} \in \mathfrak{M}\left(\pi_{1}\left(C_{0}\right), \operatorname{GL}\left(\mathbb{S}_{\mu}\left(V_{1}\right)\right)\right)^{\text {ss }}$. Now we consider the map

$$
\phi_{\mu}: \mathfrak{M}\left(\pi_{1}\left(C_{0}\right), G_{1}\right)^{\mathrm{ss}} \longrightarrow \mathfrak{M}\left(\pi_{1}\left(C_{0}\right), \mathrm{GL}\left(\mathbb{S}_{\mu}\left(V_{1}\right)\right)\right)^{\mathrm{ss}}
$$

By Simpson's main theorem in [22], there exist Hermitian Yang-Mills metrics on poly-stable Higgs bundles on $C$ with logarithmic poles of the Higgs field on $C \cap Z$. The $\mathbb{C}^{\times}$-action can be defined on both spaces of semi-simple representations on $C_{0}$ via a Hermitian Yang-Mills metric on $(\bar{E}, t \bar{\theta})$. Applying the same arguments as above to the compact case, we show that the pullback of $\left(\bar{E}_{1}, \bar{\theta}_{1}\right)$ to $C_{0}$ is a 
fixed point of the $\mathbb{C}^{\times}$-action. If we choose $C_{0}$ sufficiently ample, then $\left(\bar{E}_{1}, \bar{\theta}_{1}\right)$ is also a fixed point of the $\mathbb{C}^{\times}$-action. Again by $\left[22,\left(\bar{E}_{1}, \bar{\theta}_{1}\right)\right.$ is a $\mathbb{C}$-PVHS on $\mathbb{V}_{1}$.

Since $\mathbb{V}^{\prime}$ is a direct factor $\mathbb{V}_{1}^{*} \otimes \mathbb{V}_{1} \otimes \mathbb{V}^{\prime}=\mathbb{V}_{1}^{*} \otimes \mathbb{V}, \mathbb{V}^{\prime}$ admits a $\mathbb{C}$-PVHS as well. The tensor product of $\mathbb{C}$-PVHS on $\mathbb{V}_{1}$ and $\mathbb{V}^{\prime}$ is a $\mathbb{C}$-PVHS on $\mathbb{V}_{1} \otimes \mathbb{V}^{\prime}$. By Deligne's uniqueness theorem on $\mathbb{C}$-PVHS on irreducible local systems, the tensor product coincides with the original $\mathbb{C}$-PVHS on $\mathbb{V}_{1} \otimes \mathbb{V}^{\prime}=\mathbb{V}$. By induction on the number of factors $k$, we conclude the proof of the theorem.

Theorem 6.4. Let $S$ be a smooth quasi-projective algebraic variety and $\mathbb{V}$ be a weight $3 \mathbb{Z}$-PVHS over $S$ which is irreducible over $\mathbb{C}$ and of quasi-unipotent local monodromies. If the Hodge numbers of $\mathbb{V}$ are $(1,9,9,1)$, then after a possible finite étale base change the connected component of the real Zariski closure of the monodromy group of $\mathbb{V}$ is one of the following:

(A) $S U(1,1) \times S O_{0}(2,8)$,

(B) $S U(3,3)$,

(C) $\operatorname{Sp}(6, \mathbb{R})$,

(D) $S p(20, \mathbb{R})$.

Proof. Let $s \in S$ be a base point of $S$ and $V$ be the fiber of $\mathbb{V}$ at $s$. Let $\tau$ : $\pi_{1}(S, s) \longrightarrow G L\left(V \otimes_{\mathbb{Z}} \mathbb{R}\right)$ be the monodromy representation of $\mathbb{V} \otimes \mathbb{R}$ and $G$ be the Zariski closure of $\tau$. So we have the factorization

$$
\tau: \pi_{1}(S, s) \rightarrow G \stackrel{\rho}{\longrightarrow} G L\left(V_{\mathbb{R}}\right)
$$

where $\rho$ is a morphism of real algebraic groups. Since $\mathbb{V}$ is of polarized and of $\mathbb{Z}$-coefficients, $G$ is semi-simple by Deligne (cf. Corollary 4.2 .9 in [4]). Since $\mathbb{V}$ is of weight 3 and the dimension of $V$ is $20, G$ is a semi-simple real Lie subgroup of $S p(20, \mathbb{R})$. Let $G^{0}$ be the connected component of $G$. The classification of $G^{0}$ consists of several steps.

Step 1. Let $\mathfrak{g}$ be the Lie algebra of $G^{0}$ and $\chi: \mathfrak{g}_{\mathbb{C}} \longrightarrow \mathfrak{s p}_{20} \mathbb{C}$ be the complexification of the differential of $\rho$. Then the pair $\left(\mathfrak{g}_{\mathbb{C}}, \chi\right)$ is one of the following list:
(1) $\left(\mathfrak{s l}(2), \Gamma_{19}\right)$
(2) $\left(\mathfrak{s o}(5), \Gamma_{03}\right)$
(3) $\left(\mathfrak{s p}(6), \Gamma_{00100}\right)$
(4) $\left(\mathfrak{s l}(6), \Gamma_{00100}\right)$
(5) $\left(\mathfrak{s p}(20), \Gamma_{1000000000}\right)$
(6) $\quad\left(\mathfrak{s l}(2) \oplus \mathfrak{s l}(2), \Gamma_{3} \otimes \Gamma_{4}\right)$

$$
\begin{array}{ll}
(7) & \left(\mathfrak{s l}(2) \oplus \mathfrak{s o}(5), \Gamma_{3} \otimes \Gamma_{10}\right) \\
(8) & \left(\mathfrak{s o}(5) \oplus \mathfrak{s l}(2), \Gamma_{01} \otimes \Gamma_{4}\right) \\
(9) & \left(\mathfrak{s o}(5) \oplus \mathfrak{s o}(5), \Gamma_{10} \otimes \Gamma_{02}\right) \\
(10) & \left(\mathfrak{s l}(2) \oplus \mathfrak{s o}(5), \Gamma_{1} \otimes \Gamma_{02}\right) \\
(11) & \left(\mathfrak{s l}(2) \oplus \mathfrak{s o}(10), \Gamma_{1} \otimes \Gamma_{10000}\right)
\end{array}
$$

Here we use the notations as given in [8]. The list results from a rather standard calculation in the representation theory of semi-simple complex Lie algebras. As $\mathfrak{g}_{\mathbb{C}}$ is semi-simple, we can write $\mathfrak{g}_{\mathbb{C}}=\bigoplus_{i=1}^{m} \mathfrak{g}_{i}$ into direct sum of simple Lie algebras. By Schur's lemma and since $\chi$ is irreducible, we have the tensor decomposition of $\chi=\otimes \chi_{i}$ into irreducible representations. Then one has particularly $\prod_{i=1}^{m} d_{i}=20$, where $d_{i}$ is the dimension of the representation space of $\chi_{i}$. It is straightforward to write down a complete list of irreducible representations of 
complex simple Lie algebras whose dimensions divide 20 . For each pair $\left(\mathfrak{g}_{\mathbb{C}}, \chi\right)$ where $\chi: \mathfrak{g}_{\mathbb{C}} \rightarrow \mathfrak{s l}(20)$ in this preliminary list, it appears in the final list, i.e. $\chi$ factors through $\mathfrak{s p}(20) \subset \mathfrak{s l}(20)$ if and only if $\wedge^{2} \chi$ contains a trivial representation. This can be easily checked by using the plethysm of semi-simple complex Lie algebras.

Step 2. Let $G_{\mathbb{C}}$ be the complexification of $G^{0}$. Then by Lemma 4.4 in [23], $G^{0}$ is a real form of $G_{\mathbb{C}}$ which is of Hodge type (see $\S 4[23$ for the definition). We shall make use of the list of all simple real Lie groups of Hodge type on Page 50 [23]. In connection with the list in Step 1, one can immediately exclude the case where $\mathfrak{g}$ is simple but $\mathfrak{g}_{\mathbb{C}}$ is not simple by Proposition 4.4.10 in [23]. Hence the number of irreducible factors in $\mathfrak{g}$ is equal to that of $\mathfrak{g}_{\mathbb{C}}$.

Step 3. We start with two factors. Namely, $G^{0}=G_{1} \times G_{2}$ with $G_{i}$ simple real Lie groups. It induces the tensor decomposition of real local systems $\mathbb{V}_{\mathbb{R}}=\mathbb{V}_{1} \otimes \mathbb{V}_{2}$. By Theorem 6.3 there exist $\mathbb{C}$-PVHS structures on $\mathbb{V}_{1}$ and $\mathbb{V}_{2}$ such that the induced PVHS on $\mathbb{V}$ coincides with the original one. It follows that the Lie groups $G_{1}$ and $G_{2}$ are also of Hodge type. By Lemma 5.5 in $[23] \mathbb{V}_{i}, i=1,2$ underlies $\mathbb{R}$-PVHS structure. Recall that the weight of $\mathbb{V}$ is three and its Hodge numbers are $1,9,9,1$. Then after a possible permutation of factors the PVHS $\mathbb{V}_{1}$ must be of weight 1 with Hodge numbers 1,1 and $\mathbb{V}_{2}$ is of weight 2 with Hodge numbers 1,8,1. This implies $G_{1}=S U(1,1)$ and $G_{2} \subseteq S O(2,8)$. This excludes immediately the case (8) of the list in Step 1. It excludes also the cases (4)-(7) by checking the dimension of representation on $\mathfrak{s l}(2)$-factor. Let us now consider the case (9). Note that the representation $\Gamma_{02}$ is simply the second wedge power of the standard representation in this case. By the list in [23] $G_{2}$ can be one of the groups $S O(5), S O(1,4)$ or $S O(2,3)$. Since $S O(5)$ is compact, it is mapped into the compact form $S O(10)$ of $S O(10, \mathbb{C})$ under $\wedge^{2}$ of the standard representation of $S O(5, \mathbb{C})$. Also one checks that under the same representation the other two real forms $S O(1,4)$ and $S O(2,3)$ are mapped into the real forms $S O(6,4)$ and $S O(4,6)$ respectively. Thus the case $(9)$ can be excluded. So it remains (10) for the non-simple case. Obviously $G_{2}=S O_{0}(2,8)$ and this gives the case (A).

Step 4. We treat the case that $\mathfrak{g}_{\mathbb{C}}$ is simple. By the list in [23, $G^{0}$ can be $S U(1,1)$ in case $(1), S O(5), S O(2,3)$ and $S O(1,4)$ in case $(2), S U(p, 6-p)$ in case $(3), S p(20, \mathbb{R})$ in case $(4)$. Note that except for $S O(1,4)$ the rest groups are of Hermitian type. For them, by consideration of weight and Hodge numbers as in Step 3 we can exclude all cases except $S U(3,3)$ and $S p(20, \mathbb{R})$, which give case (B) and (C) respectively. Finally one can check directly that the real form $S O(1,4)$ of $S O(5, \mathbb{C})$ does not map into the split form $S p(20, \mathbb{R})$ of $S p(20, \mathbb{C})$ under the third wedge power. For this, one can consult for example Example 3, $\S 7$ in [20]. This completes the classification.

Theorem 6.5. Let $f: \mathcal{X} \rightarrow S$ be a good family of $\mathfrak{M}_{C Y}$ and $\mathbb{V}$ be the associated weight $3 \mathbb{Z}-P V H S$. Let $s \in S$ be a base point and let

$$
\tau: \pi_{1}(S, s) \rightarrow S p(20, \mathbb{R})
$$


be the monodromy representation associated to $\mathbb{V} \otimes \mathbb{R}$. Then the image of $\tau$ is Zariski dense in $\operatorname{Sp}(20, \mathbb{R})$.

Proof. Let $G$ be the real Zariski closure of $\tau$. Note that the Zariski dense property is not changed under a finite étale base change. By Theorem 6.4 it is to show for $\mathbb{V}$ in the statement the connected component $G^{0}$ of $G$ can not be the case (A) or (B). Assume the contrary and we shall deduce a contradiction in the following. Note that by the proof of Theorem 6.4, the inclusion $G \rightarrow S p(20, \mathbb{R})$ comes up with a uniquely determined morphism of real algebraic groups $\rho: G \rightarrow S p(20, \mathbb{R})$, which restricts to $\rho_{0}$ on $G^{0}$. Actually $\rho_{0}$ has already appeared in Proposition 3.3 implicitly. It is easy to verify that $\rho_{0}$ in each case maps the maximal compact group $K^{0}$ of $G^{0}$ into the compact subgroup $U(1) \times U(9)$ of $S p(20, \mathbb{R})$. Let $\Gamma$ be the monodromy group of $\tau$ and $\Gamma_{0}=\rho^{-1}(\Gamma) \subset G$. So we get a factorization

$$
\tau: \pi_{1}(S, s) \stackrel{\tau_{0}}{\longrightarrow} \Gamma_{0} \stackrel{\rho}{\longrightarrow} \Gamma .
$$

This gives a factorization of the period map of $\mathbb{V}$

$$
\phi: S \stackrel{j}{\longrightarrow} \Gamma_{0} \backslash G / K \stackrel{\psi}{\longrightarrow} \Gamma \backslash S p(20, \mathbb{R}) / U(1) \times U(9)
$$

where $K$ is the maximal compact group of $G$. Since $S$ is connected, the morphism $j$ factors though $\Gamma_{0}^{\prime} \backslash G^{0} / K^{0} \subset \Gamma_{0} \backslash G / K$ for $\Gamma_{0}^{\prime}=\Gamma_{0} \cap G^{0}$. Thus we arrive at the factorization in Definition 3.1. Since $\rho_{0}: G^{0} \rightarrow S p(20, \mathbb{R})$ gives rise to the canonical PVHS, the factorization contradicts with the assertion of Theorem 6.1. The proof is completed.

Corollary 6.6. The special Mumford-Tate group of a general member in $\mathfrak{M}_{C Y}$ is $\operatorname{Sp}(20, \mathbb{Q})$.

Proof. Let $f: \mathcal{X} \rightarrow S$ be a good family for $\mathfrak{M}_{C Y}$. Let $\mathbb{V}$ be the associated weight 3 $\mathbb{Q}$-PVHS of $f$ and $\tau: \pi_{1}(S, s) \rightarrow S p(20, \mathbb{Q})$ be the monodromy representation. By Deligne and Schoen (see for example Lemma 2.4 [31]), the connected component of the $\mathbb{Q}$-Zariski closure of the monodromy group is a normal subgroup of the special Mumford-Tate group $H g(\mathbb{V})$ of $\mathbb{V}$, which is equal to the special MumfordTate group of a general closed fiber of $f$. By Theorem 6.5 the $\mathbb{Q}$-Zariski closure of the monodromy group of $\mathbb{V}$ has to be the whole symplectic group $\operatorname{Sp}(20, \mathbb{Q})$, the corollary follows since the moduli map of $f$ is dominant.

Theorem 6.7. Let $\mathfrak{H}_{C Y}$ be the hyperelliptic locus of $\mathfrak{M}_{C Y}$ and $\mathfrak{H}$ be any subvariety of $\mathfrak{M}_{C Y}$ which strictly contains $\mathfrak{H}_{C Y}$. Let $f: \mathcal{X} \rightarrow S$ be a good family of $\mathfrak{M}_{C Y}$ whose moduli map $S \rightarrow \mathfrak{M}_{C Y}$ is dominant over $\mathfrak{H}$. Then the restriction of $\mathbb{V}$ to the inverse image of $\mathfrak{H}$ does not factor through $\left(D_{3}^{I I I}, \wedge^{3}\right)$.

Proof. We first prove the statement for the good family $f_{0}$ over $\mathfrak{M}_{A R}$. Assume that an extension $\mathfrak{H}$ for $f_{0}$ as in the theorem does exist. We can assume $\operatorname{dim} \mathfrak{H}=6$ without loss of generality. Let $\widetilde{\mathfrak{H}}$ be the inverse image of $\mathfrak{H}$ in $\mathfrak{M}_{A R}$ and $f_{0}^{\prime}=\left.f_{0}\right|_{\mathfrak{H}}$ the restriction of $f_{0}$ to $\widetilde{\mathfrak{H}} \subset \mathfrak{M}_{A R}$. Let $\phi$ be the period map of $f_{0}$ and $\phi^{\prime}$ that of $f_{0}^{\prime}$. By assumption one has the factorization

$$
\phi^{\prime}: \widetilde{\mathfrak{H}} \stackrel{j}{\longrightarrow} \Gamma_{0} \backslash S p(6, \mathbb{R}) / U(3) \stackrel{\wedge^{3}}{\longrightarrow} \Gamma \backslash S p(20, \mathbb{R}) / U(1) \times U(9) .
$$


By Corollary 2.2 and the local Torelli theorem for CY manifolds, $\phi$ is étale over its image. So is the restriction $\phi^{\prime}$. Because $\Gamma_{0} \backslash S p(6, \mathbb{R}) / U(3)$ is of six dimensional, $j$ is then étale. We derive the contradiction at the special point $s_{0} \in \widetilde{\mathfrak{H}}_{C Y} \subset \widetilde{\mathfrak{H}}$. We denote by $\mathcal{C}_{1}$ the first characteristic subvariety of $f_{0}$ and $\mathcal{C}_{1}^{\prime}$ that of $f_{0}^{\prime}$. By Proposition 4.6 (ii), the fiber $\left(\mathcal{C}_{1}^{\prime}\right)_{s_{0}}$ as subvariety of $\mathbb{P}\left(\mathcal{T}_{\widetilde{\mathfrak{H}}, s_{0}}\right)$ is isomorphic to the $\mathbb{P}^{2}$ into $\mathbb{P}^{5}$ via the Veronese embedding. In particular $\left(\mathcal{C}_{1}^{\prime}\right)_{s_{0}}$ is reduced. On the other hand, by Lemma 4.3 we know that

$$
\left(\mathcal{C}_{1}^{\prime}\right)_{s_{0}}=\left(\left(\mathcal{C}_{1}\right)_{s_{0}} \cap \mathbb{P}\left(\mathcal{T}_{\widetilde{\mathfrak{H}}, s_{0}}\right)\right)^{\text {red }}
$$

where the scheme-theoretical intersection of the right hand side is taken in the projective space $\mathbb{P}\left(\mathcal{T}_{\mathfrak{M}_{A R}, s_{0}}\right)$. Now by Proposition [5.6, $\left(\mathcal{C}_{1}\right)_{s_{0}}$ is two dimensional and has two irreducible components which are not contained in any linear projective subspace of dimension $\leq 6$. It follows that $\left(\left(\mathcal{C}_{1}\right)_{s_{0}} \cap \mathbb{P}\left(\mathcal{T}_{\widetilde{\mathfrak{H}}, s_{0}}\right)\right)^{\text {red }}$ is of dimension $\leq 1$. A contradiction. Thus such an extension $\mathfrak{H}$ for $f_{0}$ does not exist.

As a consequence we get the maximal property of $\mathfrak{H}_{C Y}$ stated in Corollary 1.5. This fact shows in turn the non-extension property for other good families in the theorem. Let $f$ be such a good family. As above we can assume $\operatorname{dim} \mathfrak{H}=6$. We put $S^{\prime}$ to be inverse image of $\mathfrak{H}$ under the moduli map $S \rightarrow \mathfrak{M}_{C Y}$. The factorization of the period map gives the morphism $j: S^{\prime} \rightarrow \Gamma_{0} \backslash S p(6, \mathbb{R}) / U(3)$ and it induces an isomorphism $\left.\mathbb{V}\right|_{S^{\prime}} \simeq j^{*} \mathbb{W}$ with $\mathbb{W}$ the canonical $\mathbb{R}$-PVHS over $\Gamma_{0} \backslash S p(6, \mathbb{R}) / U(3)$. Let $\mathbb{V}_{\mathbb{Q}}$ be the $\mathbb{Q}$-PVHS associated to $f$. The canonical PVHS $\mathbb{W}$ over $\Gamma_{0} \backslash S p(6, \mathbb{R}) / U(3)$ has also a natural $\mathbb{Q}$-structure $\mathbb{W}_{\mathbb{Q}}$ such that over the points in $\mathfrak{H}_{C Y}$ the isomorphism between $\mathbb{V}$ and $j^{*} \mathbb{W}$ is defined over $\mathbb{Q}$. Thus we have actually isomorphism $\left.\left(\mathbb{V}_{\mathbb{Q}}\right)\right|_{S^{\prime}} \simeq j^{*} \mathbb{W}_{\mathbb{Q}}$. This implies that the special Mumford-Tate group of a general closed point in $S^{\prime}$ and hence in $\mathfrak{H}$ is contained in $S p(6, \mathbb{Q})$. This contradicts with Corollary 1.5 .

Acknowledgements: The major part of this work was done during an academic visit of the second named author to the Department of Mathematics of the University of Mainz in 2006. He would like to express his hearty thanks to the hospitality of the faculty, especially Stefan Müller-Stach. We also thank Eckart Viehweg for his interest and several helpful conversations about this work.

\section{REFERENCES}

[1] Allcock, D.; Carlson, J.; Toledo, D.; The complex hyperbolic geometry of the moduli space of cubic surfaces. J. Algebraic Geometry. 11 (2002), no. 4, 659-724.

[2] Borcherds, R; The moduli space of Enriques surfaces and the fake monster Lie superalgebra, Topology 35, 699-710, 1996.

[3] Cynk, S.; van Straten, D.; Infinitesimal deformations of double covers of smooth algebraic varieties. Math. Nachr. 279 (2006), no. 7, 716-726.

[4] Deligne, P.; Théorie de Hodge II, Publ.Math.I.H.E.S. 40(1971), 5-57.

[5] Deligne, P.; Un théorème de finitude pour la monodromie, Discrete Groups in Geometry and Analysis, Birkhauser 1987, 1-19.

[6] Deligne, P.; Variétés de Shimura: Interpretation modulaire, et techniques de construction de modèles canoniques, Proceedings of Symposia in Pure Mathematics 33 (1979), part 2, 247-290. 
[7] Deligne, P.; Mostow, G.D.; Monodromy of hypergeometric functions and non-lattice integral monodromy, Publ. Math. I.H.E.S., Tome 63, 5-89, 1986.

[8] Fulton, W.; Harris, J.; Representation theory, A first course, GTM 129.

[9] Gerkmann, R.; Sheng, M.; Zuo, K.; Computational details on the disproof of modularity, arXiv:0709.1054, 2007.

[10] Griffiths, P.; Topics in transcendental algebraic geometry. Annals of Mathematics Studies (106), Princeton University Press, 1984.

[11] Griffiths, P.; Hodge Theory and Geometry, Bull. London Math. Soc. 36 (2004) 721-757.

[12] Gross, B.; A remark on tube domains. Mathematical Research Letters 1, 1-9, 1994.

[13] Jost, J.; Zuo, K.; Harmonic maps and $\mathrm{Sl}(r, C)$-representations of fundamental groups of quasiprojective manifolds. J. Algebraic Geometry. 5 (1996), no. 1, 77-106.

[14] Mochizuki, T.; Asymptotic behaviour of tame nilpotent harmonic bundles with trivial parabolic structure. J. Differential Geometry. 62 (2002), no. 3, 351-559.

[15] Mok, N.; Uniqueness theorems of Hermitian metrics of seminegative curvature on quotients of bounded symmetric domains. Annals of Mathematics. 125(1987), No.1, 105-152.

[16] Mok, N.; Metric rigity theorems on Hermitian locally symmetric manifolds, Series in Pure Mathmatics, Vol.6, World Scientific Publishing Co., Inc., Teaneck, NJ, 1989.

[17] Matsumoto, K.; Sasaki, T.; Yoshida, M.; The monodromy of the period map of a 4-parameter family of K3 surfaces and the hypergeometric function of type (3,6), International Journal of Mathematics, Vol 3, No.1, 1-164, 1992.

[18] Moeller, M.; Viehweg, E.; Zuo, K.; Stability of Hodge bundles and a numerical characterization of Shimura varieties, arXiv AG/07063462.

[19] Nagel, J.; The image of the Abel-Jacobi map for complete intersections, Ph.D Thesis, Rijksuniversiteit Leiden 1997.

[20] Onishchik, L.; Lectures on real semisimple Lie algebras and their representations, ESI Lectures in Mathematics and Physics. European Mathematical Society (EMS), Zürich, 2004.

[21] Peters, C.; Steenbrink, J.; Mixed Hodge structures, Ergebnisse der Mathematik und ihrer Grenzgebiete. 3. Folge 52, Springer-Verlag, Berlin, 2008.

[22] Simpson, C.; Harmonic bundles on noncompact curves. J. Amer. Math. Soc. 3 (1990), no. 3, 713-770.

[23] Simpson, C.; Higgs bundles and local systems, Publ. Math. I.H.E.S., No.75, 5-95, 1992.

[24] Simpson, C.; Moduli of representations of the fundamental group of a smooth projective varieites II, Publ. Math. I.H.E.S., No.80, 5-79, 1994.

[25] Sasaki, T.; Yamaguchi, K.; Yoshida, M.; On the ridity of differential systems modelled on Hermitian symmetric spaces and disproofs of a conjecture concerning modular interpretations of confinguration spaces. Advanced Studies in Pure Mathematics 25, 318-354, 1997.

[26] Sheng, M.; On the geometric realizations of Hermitian symmetric domains, Ph.D Thesis, The Chinese University of Hong Kong, 2006.

[27] Sheng, M.; Zuo, K.; Polarized variation of Hodge structures of $C Y$ type and characteristic subvarieties over bounded symmetric domains, To appear in Mathematische Annalen.

[28] Terasoma, T; Complete intersections of hypersurfaces - the Fermat case and the quadric case, Japan. J. Math. 14, No. 2, 309-384, 1988.

[29] Terasoma, T; Infinitesimal variation of Hodge structures and the weak global Torelli theorem for complete intersections, Annals of Mathematics, Vol. 132, No. 2, 213-225, 1990.

[30] Viehweg, E.; Quasi-projective moduli for polarized manifolds. Ergebnisse der Mathematik und ihrer Grenzgebiete (3), 30, Springer-Verlag, Berlin, 1995.

[31] Viehweg, E.; Zuo, K.; A characterization of certain Shimura curves in the moduli stack of abelian varieties, J. Differential Geometry, 66, No.2, 233-287, 2004. 
[32] Viehweg, E.; Zuo, K.; Arakelov inequalities and the uniformization of certain rigid Shimura varieties, J. Differential Geom. 77 (2007), no. 2, 291-352.

[33] Zucker,S; Locally Homogenous Variations of Hodge Structures, Enseign. Math. 27, No. 3-4, 243-276, 1982.

Universität Mainz, Fachbereich 8 (Mathematik), 55099 Mainz, Germany

E-mail address: ralf.gerkmann@math.lmu.de

East China Normal University, Dep. of Mathematics, 200062 Shanghai, P.R. China

E-mail address: msheng@math.ecnu.edu.cn, sheng@uni-mainz.de

Universität Mainz, Fachbereich 8 (Mathematik), 55099 Mainz, Germany

E-mail address: straten@mathematik.uni-mainz.de

Universität Mainz, Fachbereich 8 (Mathematik), 55099 Mainz, Germany

E-mail address: zuok@uni-mainz.de 